\title{
The disjoint $m$-flower intersection problem for latin squares
}

\author{
James G. Lefevre \\ School of Mathematics and Physics, University of Queensland \\ Brisbane, QLD, 4072, Australia \\ jgl@maths.uq.edu.au \\ Thomas A. McCourt \\ School of Mathematics and Physics, University of Queensland \\ Brisbane, QLD, 4072, Australia \\ tom.mccourt@uqconnect.edu.au \\ Submitted: Sep 1, 2010; Accepted: Jan 18, 2011; Published: Feb 21, 2011 \\ Mathematics Subject Classification: 05B15
}

\begin{abstract}
An $m$-flower in a latin square is a set of $m$ entries which share either a common row, a common column, or a common symbol, but which are otherwise distinct. Two $m$-flowers are disjoint if they share no common row, column or entry. In this paper we give a solution of the intersection problem for disjoint $m$-flowers in latin squares; that is, we determine precisely for which triples $(n, m, x)$ there exists a pair of latin squares of order $n$ whose intersection consists exactly of $x$ disjoint $m$-flowers.
\end{abstract}

\section{Introduction}

Intersection problems for latin squares were first considered by $\mathrm{Fu}$ [10]. Since then the area has been extensively investigated, see [6] for a survey of results up until 1990. Subsequent results can be found in [7], [8], [1], [3] and [9].

Intersection problems between pairs of Steiner triple systems were first considered by Lindner and Rosa [12]. Subsequently, the intersection problem, between pairs of Steiner triple systems, $\left(V, \mathcal{V}_{1}\right)$ and $\left(V, \mathcal{V}_{2}\right)$, in which the intersection of $\mathcal{V}_{1}$ and $\mathcal{V}_{2}$ is composed of a number of isomorphic copies of some specified partial triple system have also been considered. Mullin, Poplove and Zhu [15] considered the case where the partial triple system in question was a triangle. Furthermore, Lindner and Hoffman [11] considered pairs of Steiner triple systems of order $v$ intersecting in a $\left(\frac{v-1}{2}\right)$-flower and some other 
(possibly empty) set of triples; Chang and Lo Faro [4] considered the same problem for Kirkman triple systems.

In [5], Chee investigated the intersection problem for Steiner triple systems in which the intersection was composed of pairwise disjoint triples. An independent and elegant solution to this problem was given by Srinivasan [16]. This result can be considered as pairs of Steiner triple systems whose intersection is composed precisely of disjoint 1-flowers.

A natural progression of the above problems is the intersection problem for pairs of latin squares or Steiner triple systems in which the intersection is composed of a number of disjoint configurations.

In this paper the intersection problem for disjoint $m$-flowers in latin squares is solved. The solution to the corresponding problem for 2-flowers in Steiner triple systems can be found in [14].

Examples labelled A.x for some integer x refer to the appendix, which is available as a separate document from http://www.combinatorics.org/Volume_18/Abstracts/v18i1p42.html

\section{Preliminaries}

Let $N=\{i \mid 0 \leq i \leq n-1\} \subset \mathbb{N} \cup\{0\}$. Let $N^{2}$ and $N^{3}$ denote, respectively, the Cartesian products $N \times N$ and $N \times N \times N$. Let $P \subset N^{3}$ such that for any pair $n_{1}, n_{2} \in N, P$ contains at most one triple of the form $\left(n_{1}, n_{2}, n_{3}\right)$, at most one triple of the form $\left(n_{1}, n_{3}, n_{2}\right)(P$ is row latin), and at most one triple of the form $\left(n_{3}, n_{1}, n_{2}\right)$ ( $P$ is column latin), for some $n_{3} \in N$. Then the set $P$ is a partial latin square. The number of triples contained in $P$ is known as the size of $P$.

For ease of understanding the ordered triple $\left(n_{1}, n_{2}, n_{3}\right)$ may be regarded as referring to the occurrence of symbol $n_{3}$ in cell $\left(n_{1}, n_{2}\right)$ of an $n \times n$ array; this cell occurs in row $n_{1}$ and column $n_{2}$. If a cell contains no symbol it is called empty. Conversely, if a cell contains a symbol it is said to be filled. For a partial latin square $P$, its shape is the set of filled cells of $P$. If in a partial latin square, $P$, there are no empty cells then $P$ is called a latin square of order $n$.

Let $L$ be a latin square of order $n$; the set of cells $\{(i, i) \mid 0 \leq i \leq n-1\}$ is denoted as the main diagonal of $L$.

A pair of partial latin squares, $\left(P_{1}, P_{2}\right)$, is called a latin biswap if the pair satisfies the following: $P_{1}$ and $P_{2}$ have the same shape; and the corresponding rows (columns) of $P_{1}$ and $P_{2}$ contain the same entries.

Note that if $P_{1}$ is contained in a latin square $L_{1}$ then $P_{2}$ is contained in the latin square $L_{2}=\left(L_{1} \backslash P_{1}\right) \cup P_{2}$; moreover, $L_{1} \backslash P_{1}=L_{2} \backslash P_{2}$.

A latin biswap, $\left(P_{1}, P_{2}\right)$, is called a latin bitrade if it satisfies the additional property that $P_{1} \cap P_{2}=\emptyset$.

A transversal, $T$, in a latin square $L$ of order $n$, (that is $T \subset L$ ) is a partial latin square which contains $n$ triples such that each element in $N$ occurs precisely once in a coordinate of a triple in $T$. 
Let $L$ be a latin square of order $n$ that contains two transversals $S$ and $T$. If the shape of $S$ has no cells in common with the shape of $T$, then $S$ and $T$ are said to be disjoint.

In the following a configuration, $P$, is an isomorphic copy of some specified partial latin square.

In a latin square, $L$, an $m$-flower is a configuration containing $m$ triples and which is of the form

$$
F=\left\{\left(x_{i}, y_{i}, z_{i}\right) \mid 1 \leq i \leq m\right\} \subseteq L \text { such that precisely one of the following holds : }
$$

for all distinct $i$ and $j: x_{i}=x_{j}, y_{i} \neq y_{j}$ and $z_{i} \neq z_{j}$ (a row-m-flower); or

for all distinct $i$ and $j: x_{i} \neq x_{j}, y_{i}=y_{j}$ and $z_{i} \neq z_{j}$ (a column-m-flower); or

for all distinct $i$ and $j: x_{i} \neq x_{j}, y_{i} \neq y_{j}$ and $z_{i}=z_{j}$ (a symbol-m-flower).

If the intersection between the row coordinates, the intersection between the column coordinates and the intersection between the symbol coordinates of two $m$-flowers are all empty then the two $m$-flowers are said to be pairwise disjoint. If a set of $k m$-flowers satisfy the property that any two are pairwise disjoint then it is said to be a set of $k$ disjoint $m$-flowers.

Consider a set of $k$ disjoint $m$-flowers. A triple in which the row, column and entry coordinates are not equal to, respectively, the row, column or entry of any of the triples in the $k$ disjoint $m$-flowers, is said to be a disjoint triple (to the $m$-flowers).

\section{Constructions for latin squares}

For each map $f$ defined in this paper, the image of all the triples in a subset $P \subset N^{3}$ under $f$ will be denoted by $f P$.

Throughout this paper the well known technique of prolongation is extensively used. This section begins by briefly discussing this technique.

Consider a latin square, $L$, of order $n$, and assume that $L$ contains a transversal $T$; then construct a latin square, $L(+1)$, of order $n+1$, as follows: $L(+1)=$

$$
(L \backslash T) \cup\{(x, y, n),(n, y, z),(x, n, z) \mid(x, y, z) \in T\} \cup\{(n, n, n)\} .
$$

If the latin square $L$ contains $k$ disjoint transversals, $T_{i}$, where $1 \leq i \leq k$, this idea can be generalised to a $k$-prolongation, yielding a latin square $L(+k)$ of order $n+k$.

Let $\zeta_{r}$ and $\zeta_{c}$ be elements of the symmetric group, $S_{k}$, acting on the set $\{i \mid 1 \leq i \leq k\}$.

Let $1 \leq k, n$ and $\mathcal{P}$ be a partial latin square of order $n+k$ in which the cells in the set $\{(i, j) \mid n \leq i, j \leq n+k-1\}$ are filled with symbols from the set $\{i \mid n \leq i \leq n+k-1\}$ and all other cells are empty; such a partial latin square is denoted as a completing square (note that such a partial latin square exists for all orders as it corresponds to a latin square of order $k$ in which each triple $(a, b, c)$ is replaced with $(a+n, b+n, c+n))$.

Let $n^{\prime}, n \in \mathbb{N}$ such that $n^{\prime} \leq n, N^{\prime}=\left\{i \mid 0 \leq i \leq n^{\prime}-1\right\}$ and $N=\{i \mid 0 \leq i \leq n\}$. Define the following maps from $N^{\prime 3}$ to $N^{3}$.

$$
\gamma_{r}^{n}:(u, v, w) \mapsto(n, v, w) ; \gamma_{c}^{n}:(u, v, w) \mapsto(u, n, w) ; \text { and } \gamma_{s}^{n}:(u, v, w) \mapsto(u, v, n)
$$


Then $L(+k)=$

$$
\left(L \backslash \bigcup_{1 \leq i \leq k} T_{i}\right) \cup \bigcup_{1 \leq i \leq k}\left(\gamma_{r}^{\zeta_{r}(i)+n-1} T_{i} \cup \gamma_{c}^{\zeta_{c}(i)+n-1} T_{i} \cup \gamma_{s}^{i+n-1} T_{i}\right) \cup \mathcal{P}
$$

is a latin square of order $n+k$. See Example A.1. This latin square is referred to as a $\left(\zeta_{r}, \zeta_{c}\right)$-k-prolongation of $L$. If $\zeta_{r}=\zeta_{c}=i d$, the identity permutation, then $L(+k)$ is simply referred to as a $k$-prolongation of $L$.

Let $j \in \mathbb{N}$ and $J=\{i \mid 0 \leq i \leq j-1\}$. Define the following maps from $J^{3}$ to $J^{3}$ for $0 \leq i, l \leq j-1$.

$$
\begin{aligned}
& i d:(u, v, w) \mapsto(u, v, w) ; \\
& \sigma_{r}^{j}:(u, v, w) \mapsto((u+1(\bmod j)), v, w) ; \\
& \sigma_{c}^{j}:(u, v, w) \mapsto(u,(v+1(\bmod j)), w) ; \\
& \sigma_{s}^{j}:(u, v, w) \mapsto(u, v,(w+1(\bmod j))) ; \\
& \sigma_{s}^{i, j}:(u, v, w) \mapsto(u, v,(w-i+1(\bmod j-i))+i) ; \\
& v_{s}^{j}:(u, v, w) \mapsto\left\{\begin{array}{ll}
(u, v,(w+1(\bmod j-1))), & 0 \leq w \leq j-2 \\
(u, v, w), & w=j-1
\end{array} ;\right. \\
& \epsilon_{s}^{j}:(u, v, w) \mapsto\left\{\begin{array}{ll}
(u, v,(w+1(\bmod j-3))), & 0 \leq w \leq j-4 \\
(u, v, w), & w=j-2 \\
(u, v, j-3), & w=j-1 \\
(u, v, j-1), & w=j-3
\end{array} ;\right. \\
& \phi_{s}^{j}:(u, v, w) \mapsto(u, v, w-2(\bmod j)) ; \\
& \delta_{i}^{j}:(u, v, w) \mapsto(i+u(\bmod j), i+w(\bmod j), i+v(\bmod j)) ; \\
& \rho_{i}^{l}:(u, v, w) \mapsto\left\{\begin{array}{ll}
(u, i, w) & \text { if } v=l \\
(u, l, w) & \text { if } v=i \\
(u, v, w) & \text { otherwise }
\end{array} ;\right. \text { and } \\
& \psi_{i}^{j}:(u, v, w) \mapsto\left\{\begin{array}{ll}
(u, v, w+1(\bmod i)), & \text { if } 2 \leq w \leq i-1 \\
(u, v, 2), & \text { if } w=0 \\
(u, v, w), & \text { otherwise }
\end{array} .\right.
\end{aligned}
$$

Let $A$ be a partial latin square of order $a$ and let $B$ be a partial latin square of order $b$. Let $f_{\alpha} \in\left\{i d, \sigma_{r}^{b}, \sigma_{c}^{b}, \sigma_{s}^{b}, \phi_{s}^{b}\right\}$ where $\alpha=(u, v, w) \in A$. Define the product of the singleton $\{\alpha\}$ and $B$ as follows; $\{\alpha\} \times f_{\alpha} B=\left\{(u b+x, v b+y, w b+z) \mid(x, y, z) \in f_{\alpha} B\right\}$. Now define the direct product of $A$ and $B$ as follows; $A \times f_{\alpha} B=\bigcup_{\alpha \in A}\left(\{\alpha\} \times f_{\alpha} B\right)$. If for all $\alpha \in A$, $f_{\alpha}=i d$, simply write $A \times B$.

Throughout this paper use will be made of the following technical lemma. 
Lemma 3.1. Let $P$ and $P^{\prime}$ be two partial latin squares, both of order $p$; let $\alpha=(u, v, w) \in$ $P$ and $\beta=\left(u^{\prime}, v^{\prime}, w^{\prime}\right) \in P^{\prime}$. Let $\Sigma \in\{\sigma, \phi\}$; let $j \in\{r, c, s\}$, with $j=s$ if $\Sigma=\phi$. Let $T$ be some transversal of order $t>2$. Finally, let $k \in \mathbb{N}$ such that $k \geq p t$.

Then

$$
\begin{aligned}
& \left(\gamma_{r}^{k}(\{\alpha\} \times T)\right) \cap\left(\gamma_{r}^{k}\left(\{\beta\} \times \Sigma_{j}^{t} T\right)\right)=\left\{\begin{array}{ll}
\gamma_{r}^{k}(\{\alpha\} \times T), & j=r, v=v^{\prime} \text { and } w=w^{\prime} \\
\emptyset, & \text { otherwise }
\end{array} ;\right. \\
& \left(\gamma_{c}^{k}(\{\alpha\} \times T)\right) \cap\left(\gamma_{c}^{k}\left(\{\beta\} \times \Sigma_{j}^{t} T\right)\right)=\left\{\begin{array}{ll}
\gamma_{c}^{k}(\{\alpha\} \times T), & j=c, u=u^{\prime} \text { and } w=w^{\prime} \\
\emptyset, & \text { otherwise }
\end{array} ;\right. \\
& \left(\gamma_{s}^{k}(\{\alpha\} \times T)\right) \cap\left(\gamma_{s}^{k}\left(\{\beta\} \times \Sigma_{j}^{t} T\right)\right)= \begin{cases}\gamma_{s}^{k}(\{\alpha\} \times T), & j=s, u=u^{\prime} \text { and } v=v^{\prime} \\
\emptyset, & \text { otherwise }\end{cases}
\end{aligned}
$$

Proof. The third statement will be proved for $\Sigma=\sigma$, the other cases follow similarly.

First let $j=s$.

Then $\{\beta\} \times \sigma_{s}^{t} T=\left\{\left(u^{\prime} t+x, v^{\prime} t+y, w^{\prime} t+(z+1(\bmod \mathrm{t}))\right) \mid(x, y, z) \in T\right\}$, hence, $\gamma_{s}^{k}\left(\{\beta\} \times \sigma_{s}^{t} T\right)=\left\{\left(u^{\prime} t+x, v^{\prime} t+y, k\right) \mid(x, y, z) \in T\right\}$.

Also, $\quad\{\alpha\} \times T=\{(u t+x, v t+y, w t+z) \mid(x, y, z) \in T\}$, so, $\quad \gamma_{s}^{k}(\{\alpha\} \times T)=\{(u t+x, v t+y, k) \mid(x, y, z) \in T\}$.

Thus,

$$
\gamma_{s}^{k}(\{\alpha\} \times T)=\gamma_{s}^{k}\left(\{\beta\} \times \sigma_{s}^{t} T\right)
$$

if and only if $u=u^{\prime}$ and $v=v^{\prime}$; otherwise the intersection of these two sets is empty.

Now consider the case where $j=c$.

Then $\{\beta\} \times \sigma_{c}^{t} T=\left\{\left(u^{\prime} t+x, v^{\prime} t+(y+1(\bmod t)), w^{\prime} t+z\right) \mid(x, y, z) \in T\right\}$, hence, $\gamma_{s}^{k}\left(\{\beta\} \times \sigma_{c}^{t} T\right)=\left\{\left(u^{\prime} t+x, v^{\prime} t+(y+1(\bmod t)), k\right) \mid(x, y, z) \in T\right\}$.

Thus,

$$
\gamma_{s}^{k}(\{\alpha\} \times T) \cap \gamma_{s}^{k}\left(\{\beta\} \times \sigma_{c}^{t} T\right)=\emptyset
$$

regardless of whether or not $u=u^{\prime}$ or $v=v^{\prime}$.

The subcase where $j=r$ follows similarly.

More often than not, when Lemma 3.1 is applied, $P=P^{\prime}$ and $\alpha=\beta$.

The following is a well known result [2].

Lemma 3.2. (Bose, Shrikhande \& Parker, [2]) For all $3 \leq n, n \neq 6$ there exists a latin square which is composed of $n$ disjoint transversals. For $n=6$ there exists a latin square that contains 4 disjoint transversals.

Extensive use will be made of the following result.

Lemma 3.3. Let $A$ be a partial latin square of order a that contains a transversal $U$. Let $B$ be a partial latin square of order $b$ that contains a transversal, $T$. Let $f_{\alpha} \in$ $\left\{i d, \sigma_{r}^{b}, \sigma_{c}^{b}, \sigma_{s}^{b}, \phi_{s}^{b}\right\}$, where $\alpha \in A$. Then $U \times f_{\alpha} T$ is a transversal in $A \times f_{\alpha} B$. 
Proof. As the rows, columns or symbols can be reordered, without loss of generality $U=\{(j, j, j) \mid 0 \leq j \leq a-1\}$.

Consider $U \times f_{\alpha} T$, note that $\left|U \times f_{\alpha} T\right|=a b$.

Now, $U \times f_{\alpha} T=\bigcup_{0 \leq j \leq a-1}\left\{(j b+x, j b+y, j b+z) \mid(x, y, z) \in f_{(j, j, j)} T\right\}$.

Note that $f_{(j, j, j)} T$ is a transversal in $f_{(j, j, j)} B$. Hence,

$$
\begin{aligned}
\bigcup_{0 \leq j \leq a-1}\left\{(j b+x) \mid(x, y, z) \in f_{(j, j, j)} T\right\} & =\bigcup_{0 \leq j \leq a-1}\left\{(j b+y) \mid(x, y, z) \in f_{(j, j, j)} T\right\} \\
& =\bigcup_{0 \leq j \leq a-1}\left\{(j b+z) \mid(x, y, z) \in f_{(j, j, j)} T\right\} \\
& =\{j b+h \mid 0 \leq h \leq b-1,0 \leq j \leq a-1\} \\
& =\{i \mid 0 \leq i \leq a b-1\} .
\end{aligned}
$$

Therefore, $U \times f_{\alpha} T$ is a transversal in $A \times f_{\alpha} B$.

\section{Solving the intersection problem}

The previous two sections have detailed the notation and constructions which will be used to provide a solution to the intersection problem for disjoint $m$-flowers in latin squares. This result is presented in Theorem 1, at the end of this section.

The necessary and sufficient conditions for the proof of Theorem 1 are covered in the following pages. To aid the reader two tables are provided; Table 1 indicates the lemmas that establish necessary conditions whilst Table 2 indicates the lemmas that establish sufficient conditions.

For ease of notation throughout this paper any set of the form $\{i \mid p \leq i \leq p-1\}$ is taken to be the empty set.

Table 1: Necessary Conditions for Theorem 1

\begin{tabular}{|l|l|}
\hline Condition & Lemmas \\
\hline \hline Maximum number of $m$-flowers & 4.1 \\
\hline General exceptions & $4.2,4.4$ \\
\hline Exceptions for pairs of latin squares of small order & $4.5,4.13$ \\
\hline
\end{tabular}

Lemma 4.1. Let $l \in \mathbb{N} \cup\{0\}, L$ be a latin square of order $n$ and $m \leq n$ then if:

$l(2 m+1) \leq n<l(2 m+1)+m ; L$ contains a maximum $3 l$ disjoint $m$-flowers;

$l(2 m+1)+m \leq n<l(2 m+1)+2 m ; L$ contains a maximum $3 l+1$ disjoint $m$-flowers;

$n=l(2 m+1)+2 m ; L$ contains a maximum $3 l+2$ disjoint $m$-flowers. 
Table 2: Sufficient Conditions for Theorem 1

\begin{tabular}{|l|l|}
\hline Condition & Lemmas \\
\hline \hline 2-flowers in pairs of latin squares of small order & 4.3 \\
\hline One $m$-flower & 4.11 \\
\hline Two disjoint $m$-flowers & 4.19 \\
\hline $\begin{array}{l}\text { Three disjoint } m \text {-flowers in pairs of latin squares of order } \\
2 m+1 \leq n \leq 3 m\end{array}$ & $4.20,4.23$ \\
\hline $\begin{array}{l}\text { Three or four disjoint } m(\geq 3) \text {-flowers in pairs of latin } \\
\text { squares of order } 3 m+1 \leq n \leq 4 m\end{array}$ & $4.24,4.25$ \\
\hline $\begin{array}{l}\text { Three, four or five disjoint } m(\geq 3) \text {-flowers in pairs of } \\
\text { latin squares of order } n=4 m+1\end{array}$ & $4.26,4.27$ \\
\hline $\begin{array}{l}\text { Three, four, five or six disjoint } m(\geq 3) \text {-flowers in pairs } \\
\text { of latin squares of order } 4 m+2 \leq n \leq 6 m+2\end{array}$ & $4.28,4.29$ \\
\hline $\begin{array}{l}\text { Seven disjoint } m(\geq 3) \text {-flowers in pairs of latin squares } \\
\text { of order } 5 m+2 \leq n \leq 6 m+2\end{array}$ & $4.32,4.33$ \\
\hline $\begin{array}{l}\text { Eight disjoint } m(\geq 3) \text {-flowers in pairs of latin squares of } \\
\text { order } 6 m+2\end{array}$ & 4.31 \\
\hline $\begin{array}{l}0 \leq h \leq 3\left\lfloor\frac{n}{2 m+1}\right\rfloor \text { disjoint } m \text {-flowers in pairs of latin } \\
\text { squares of order } n \geq 6 m+3\end{array}$ & $4.34,4.35$ \\
\hline $\begin{array}{l}l \geq 3 ; 3 l+1 \text { disjoint } m \text {-flowers in pairs of latin squares } \\
\text { of order } l(2 m+1)+m \leq n \leq l(2 m+1)+2 m\end{array}$ & $4.36,4.37$, \\
\hline $\begin{array}{l}l \geq 3 ; 3 l+2 \text { disjoint } m \text {-flowers in pairs of latin squares } \\
\text { of order } n=l(2 m+1)+2 m\end{array}$ & 4.38 \\
\hline
\end{tabular}

Proof. Assume that there are $k=k_{1}+k_{2}+k_{3}$ disjoint $m$-flowers in a latin square, $L$, of order $n$, such that there are $k_{1}$ row- $m$-flowers; $k_{2}$ column- $m$-flowers; and $k_{3}$ symbol- $m$ flowers.

Thus, in $L$ the $k$ disjoint $m$-flowers contain, $k_{1}+m k_{2}+m k_{3}$ distinct rows, $m k_{1}+k_{2}+m k_{3}$ distinct columns, and $m k_{1}+m k_{2}+k_{3}$ distinct symbols.

Hence, $n \geq k_{1}+m\left(k_{2}+k_{3}\right)=k_{1}+m\left(k-k_{1}\right)$. Without loss of generality, let $k_{1} \leq k_{2} \leq$ $k_{3}$; this implies that $k_{1} \leq\left\lfloor\frac{k}{3}\right\rfloor$. Thus, $n \geq\left\lfloor\frac{k}{3}\right\rfloor+m\left(k-\left\lfloor\frac{k}{3}\right\rfloor\right)$, and the result follows.

Lemma 4.2. There does not exist a pair of latin squares of order $2 m+1$ whose intersection is precisely three disjoint $m$-flowers.

Proof. In order for a latin square of order $2 m+1$ to contain three disjoint $m$-flowers, one $m$-flower is required to be a symbol- $m$-flower, one to be a row- $m$-flower and one to be a column- $m$-flower.

Consider a latin square $L$ of order $2 m+1$ that contains a row- $m$-flower in row $i$ that is disjoint to a column- $m$-flower in column $j$. Both of these $m$-flowers contain $m$ symbols and all these $2 m$ symbols must be distinct. Hence, there is only one choice for the symbol 
contained in cell $(i, j)$.

In [13] latin squares of small orders are provided that establish the following result.

Lemma 4.3. (McCourt, [13]) There exist pairs of latin squares of order $n$ that intersect in $x$ 2-flowers, where:

$$
\begin{aligned}
& n=5 \text { and } x=2 ; \text { or } n=6 \text { and } 2 \leq x \leq 3 ; \text { or } n=7 \text { and } 2 \leq x \leq 4 ; \text { or } \\
& n=8 \text { and } 2 \leq x \leq 4 ; \text { or } n=9 \text { and } 2 \leq x \leq 5 ; \text { or } n=10 \text { and } 2 \leq x \leq 6 ; \text { or } \\
& n=11 \text { and } 2 \leq x \leq 6 ; \text { or } n=12 \text { and } 2 \leq x \leq 7 ; \text { or } n=13 \text { and } 2 \leq x \leq 7 ; \text { or } \\
& n=14 \text { and } 2 \leq x \leq 8
\end{aligned}
$$

\subsection{One $m$-flower}

In this section pairs of latin squares of order $n$ that intersect precisely in one $m$-flower, where $m \leq n$, and no other triples will be constructed. Without loss of generality, the $m$-flower can be considered to be a symbol- $m$-flower. By permuting the rows, columns or symbols the symbol- $m$-flower can be placed along $m$ cells of the main diagonal, and the common symbol may be chosen to be zero.

Lemma 4.4. No pair of latin squares of order $n$ can intersect in an $(n-1)$-flower.

Proof. Consider a partial latin square, $P$, of order $n$ that contains the triples in the set $\{(i, i, 0) \mid 0 \leq i \leq n-2\}$ and the triple $(n-1, n-1, x)$, where $x \neq 0$. For $P$ to be completed the symbol 0 must occur once more in the latin square, however there is no cell in which it can be placed without invalidating the row or column latin property.

Thus, the set of possible values of $m$ such that there exists a pair of latin squares of order $n$ that intersect precisely in one $m$-flower is the set $J S(n)=\{0,1,2, \ldots, n-2, n\}$. The set of achievable values of $m$ such that there exists a pair of latin squares of order $n$ that intersect precisely in one $m$-flower will be denoted by $I S(n)$.

Let $L$ be a latin square of order $n$. Then $\sigma_{r}^{n} L$ is a latin square of order $n$ such that $L$ and $\sigma_{r}^{n} L$ have no triples in common. Hence, $0 \in I S(n)$. Also, $\mathrm{Fu}$ in [10] showed that for all $n \geq 4$ there exists a pair of latin squares that intersect precisely in one triple. Furthermore, in [10] $\mathrm{Fu}$ showed that two latin squares of order three can not intersect precisely in one triple. Hence, the following result has been established.

Lemma 4.5. (Fu, [10]) For all $4 \leq n, 0,1 \in I S(n)$. Furthermore $1 \notin I S(3)$.

Now, pairs of latin squares of order $n$ to establish the contents of $I S(n)$ will be constructed. The construction used is recursive and [13] provides the necessary "ingredient" latin squares, of orders less than 8, required for the recursion to take effect.

By inspection no pair of latin squares of order two intersect in precisely one 2-flower. This coupled with Lemma 4.5 and the intersections between latin squares given in [13] yields the following result.

Lemma 4.6. For pairs of latin squares of order $i$, where $2 \leq i \leq 7$; 


$$
\begin{aligned}
& I S(2)=\{0\} ; I S(3)=\{0,3\} ; I S(4)=J S(4) ; I S(5)=J S(5) ; I S(6)=J S(6) ; \text { and } \\
& I S(7)=J S(7) .
\end{aligned}
$$

The construction used for latin squares of order greater than or equal to eight splits into two cases.

\subsubsection{Case A: $n=2 k$}

For pairs of latin squares of order $n=2 k \geq 8$ a simple doubling construction is used.

Consider the latin square $A=\{(0,0,0),(0,1,1),(1,0,1),(1,1,0)\}$ of order two. Let $m_{1}, m_{2} \in\{0,1,2, \ldots, k-2, k\}$. Now, assume there exists a pair of latin squares, $\left\{U_{1}, U_{2}\right\}$, of order $k$ whose intersection is the set of triples $\left\{(i, i, 0) \mid 0 \leq i \leq m_{1}-1\right\}$ (a symbol- $m_{1}$ flower). Similarly, assume there exists a pair of latin squares, $\left\{V_{1}, V_{2}\right\}$, of order $k$ whose intersection is the set of triples $\left\{(i, i, 0) \mid 0 \leq i \leq m_{2}-1\right\}$ (a symbol- $m_{2}$-flower).

A pair of latin squares, $\left\{L_{1}, L_{2}\right\}$, of order $2 k=n$ that intersect precisely in one $\left(m_{1}+m_{2}\right)$-flower is now constructed. First, let

$$
L_{1}=\{(0,0,0)\} \times U_{1} \cup\{(0,1,1)\} \times U_{1} \cup\{(1,0,1)\} \times U_{1} \cup\{(1,1,0)\} \times V_{1} .
$$

Now, let

$$
L_{2}=\{(0,0,0)\} \times U_{2} \cup\{(0,1,1)\} \times \sigma_{s}^{k} U_{1} \cup\{(1,0,1)\} \times \sigma_{s}^{k} U_{1} \cup\{(1,1,0)\} \times V_{2} .
$$

The intersection of $L_{1}$ and $L_{2}$ is the set of triples $\{(i, i, 0),(k+j, k+j, 0) \mid 0 \leq i \leq$ $\left.m_{1}-1,0 \leq j \leq m_{2}-1\right\}$, an $\left(m_{1}+m_{2}\right)$-flower. Note that the rows or columns of $L_{1}$ and $L_{2}$ can be permuted so that the intersection of $L_{1}$ and $L_{2}$ (the $\left(m_{1}+m_{2}\right)$-flower) is composed of the set of triples $\left\{(i, i, 0) \mid 0 \leq i \leq m_{1}+m_{2}-1\right\}$. Thus, the following result has been established.

Lemma 4.7. Assume that $4 \leq k$ and $I S(k)=J S(k)$. Let $n=2 k$, then $I S(n)=J S(n)$.

\subsubsection{Case B: $n=2 k+1$}

One $m$-flower: $m \in\{i \mid 0 \leq i \leq k-2\} \cup\{k\}$

A pair of latin squares, $\left\{L_{1}, L_{2}\right\}$, of order $2 k+1 \geq 9$ that intersect precisely in one $m$-flower, where $m \in\{i \mid 0 \leq i \leq k-2\} \cup\{k\}$ will now be constructed.

Let $m \in\{i \mid 0 \leq i \leq k-2\} \cup\{k\}$. Also, assume there exists a pair of latin squares, $\left\{U_{1}, U_{2}\right\}$, of order $k \geq 4$ whose intersection is composed precisely of the triples $\{(i, i, 0) \mid 0 \leq i \leq m-1\}$ (a symbol- $m$-flower).

Consider the following partial latin squares.

$$
\begin{gathered}
A(2 k+1)=\{(i, j,(i+j(\bmod k+1))+k),(j, i,(i+j(\bmod k+1))+k), \\
(j, j,(j-1(\bmod k+1))+k) \mid 0 \leq i \leq k-1, k \leq j \leq 2 k\} . \\
B(2 k+1)=\{(i, j, i-j(\bmod k)) \mid k \leq i<j \leq 2 k\} \cup\{(i, j, i-j-1(\bmod k)) \mid k \leq j< \\
i \leq 2 k\} .
\end{gathered}
$$




\section{Example 4.1.}
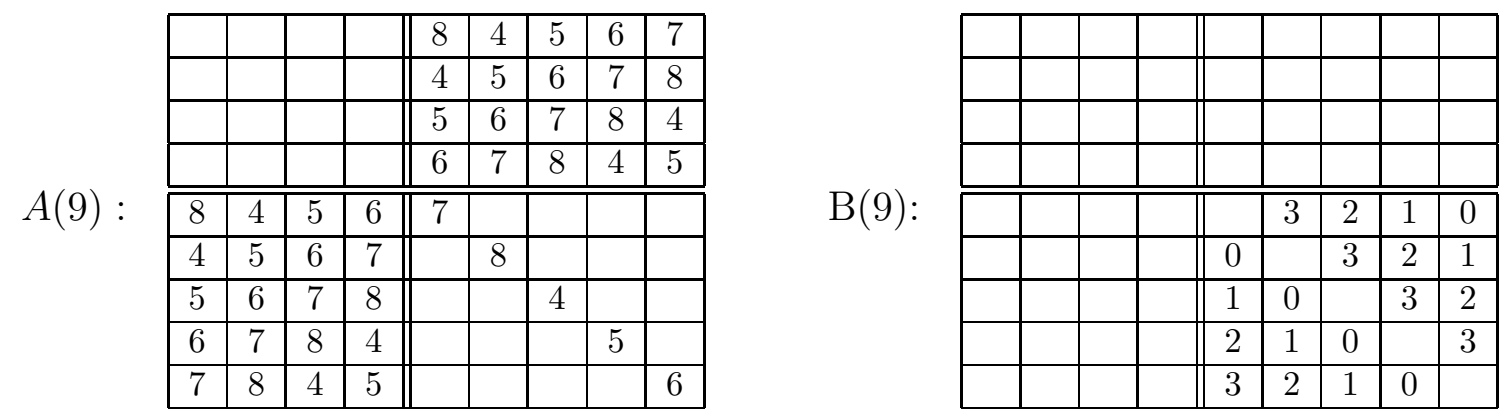

Construct the latin square $L_{1}=U_{1} \cup A(2 k+1) \cup B(2 k+1)$ of order $2 k+1$. Now, construct the latin square $L_{2}=U_{2} \cup \sigma_{s}^{k, 2 k+1} A(2 k+1) \cup \sigma_{s}^{k} B(2 k+1)$ of order $2 k+1$.

The intersection of $L_{1}$ and $L_{2}$ is the set of triples $\{(i, i, 0) \mid 0 \leq i \leq m\}$, an $m$-flower. Hence, the following result has been established.

Lemma 4.8. Assume that $4 \leq k$ and $I S(k)=J S(k)$. Let $n=2 k+1$, then $\{i \mid 0 \leq i \leq$ $k-2\} \cup\{k\} \subseteq I S(n)$.

\section{One $(k-1)$-flower}

Now, a pair of latin squares, $\left\{L_{1}, L_{3}\right\}$, of order $2 k+1 \geq 9$ that intersect precisely in one $(k-1)$-flower will be constructed. Again, assume there exists a pair of latin squares of order $k$ whose intersection is precisely one $k$-flower. Using the above construction, a pair of latin squares, $\left\{L_{1}, L_{2}\right\}$, is constructed, that intersect precisely in one $k$-flower. Now construct the latin square $L_{3}=\rho_{k-2}^{2 k} L_{2}$ (the mapping $\rho_{k-2}^{2 k}$ simply swaps column $k-2$ with column $2 k$ in $L_{2}$ ).

This yields a pair of latin squares, $\left\{L_{1}, L_{3}\right\}$, of order $2 k+1$, whose intersection is precisely one $(k-1)$-flower. Hence, the following result has been established.

Lemma 4.9. Assume that $4 \leq k$ and $k \in I S(k)$. Let $n=2 k+1$, then $k-1 \in I S(n)$.

One $m$-flower: $m \in\{i \mid k+1 \leq i \leq 2 k-1\} \cup\{2 k+1\}$

Next, a pair of latin squares, $\left\{L_{1}, L_{2}\right\}$, of order $2 k+1$ that intersect precisely in one $m$-flower, where $m \in\{i \mid k+1 \leq i \leq 2 k-1\} \cup\{2 k+1\}$ will be constructed.

Let $m_{1} \in\{j \mid 0 \leq j \leq k-2\} \cup\{k\}$. Assume there exists a pair of latin squares, $\left\{U_{1}, U_{2}\right\}$, of order $k$ whose intersection is composed precisely of the triples $\{(i, i, 0) \mid 0 \leq$ $\left.i \leq m_{1}-1\right\}$ (a symbol- $m_{1}$-flower).

Consider the following partial latin squares.

$$
\begin{gathered}
C(2 k+1)=\{(i, j,(i+j+1(\bmod k+1))+k) \mid 0 \leq i \leq k-1, k \leq j \leq 2 k\} . \\
D(2 k+1)=\{(i, j,(i+j(\bmod k+1))+k) \mid k \leq i \leq 2 k, 0 \leq j \leq k-1\} . \\
E_{1}(2 k+1)=\{(i, j, i-j-1(\bmod k+1)) \mid k \leq i, j \leq 2 k, i \neq j,(i(\bmod k+1))+k \neq j\} . \\
F_{1}(2 k+1)=\{(i,(i(\bmod k+1))+k,(i-1(\bmod k+1))+k) \mid k \leq i \leq 2 k\} . \\
G(2 k+1)=\{(i, i, 0) \mid k \leq i \leq 2 k\} .
\end{gathered}
$$




\section{Example 4.2.}

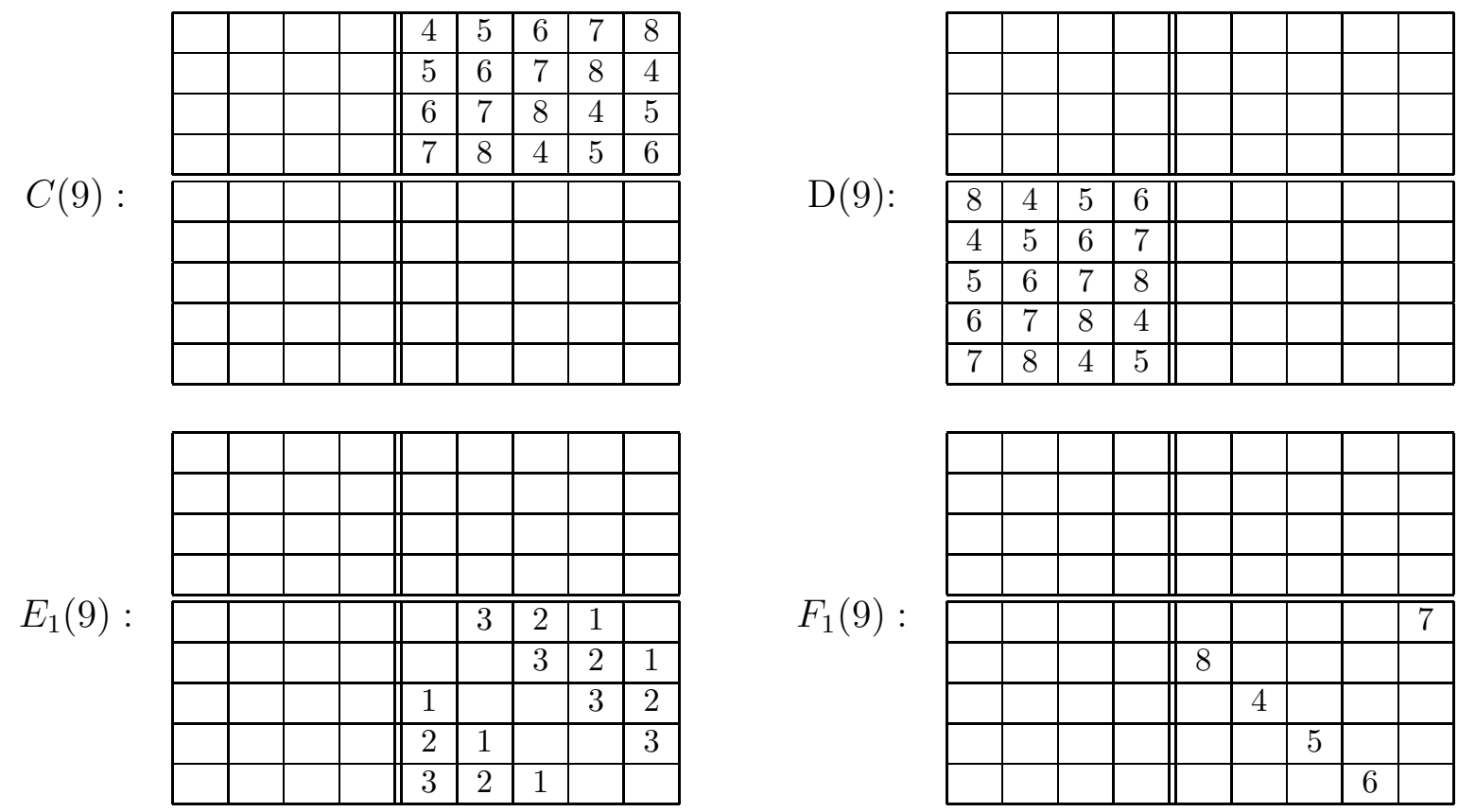

Construct the following latin square of order $2 k+1$;

$$
L_{1}=U_{1} \cup C(2 k+1) \cup D(2 k+1) \cup E_{1}(2 k+1) \cup F_{1}(2 k+1) \cup G(2 k+1) .
$$

Consider the following partial latin squares.

$$
\begin{gathered}
\left.E_{2}(2 k+1)=\{(i, j, i-j(\bmod k+1)) \mid k \leq i, j \leq 2 k, i \neq j,(i+2(\bmod k+1))+k \neq j\}\right\} . \\
F_{2}(2 k+1)=\{(i,(i+2(\bmod k+1))+k, i) \mid k \leq i \leq 2 k\} .
\end{gathered}
$$

\section{Example 4.3.}
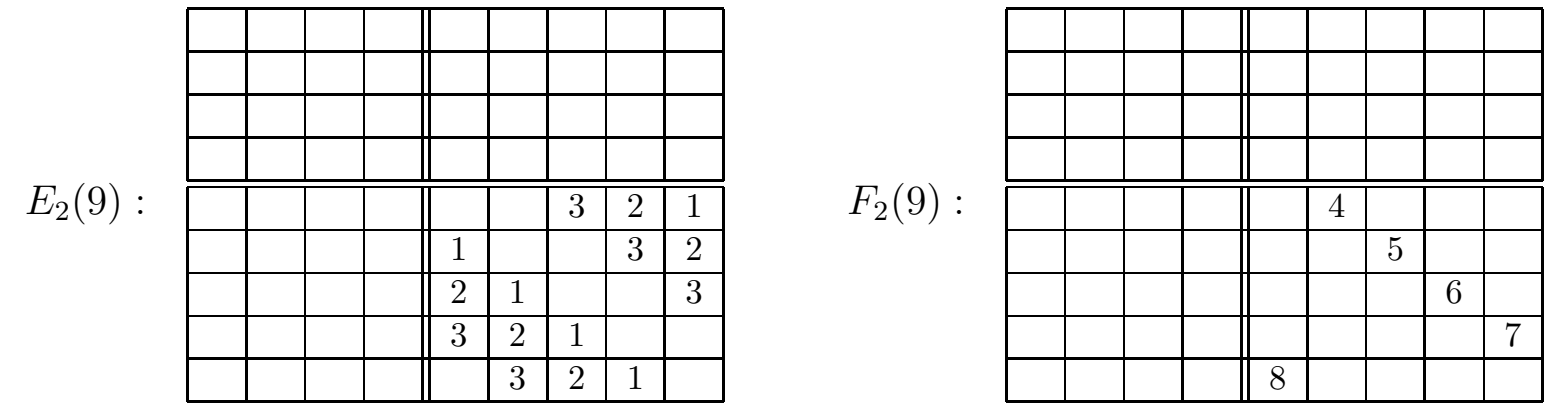

Now construct the following latin square of order $2 k+1$;

$L_{2}=U_{2} \cup \sigma_{r}^{k} C(2 k+1) \cup \sigma_{s}^{k, 2 k+1} \sigma_{s}^{k, 2 k+1} D(2 k+1) \cup E_{2}(2 k+1) \cup F_{2}(2 k+1) \cup G(2 k+1)$.

The intersection of $L_{1}$ and $L_{2}$ is the set of triples $\left\{(i, i, 0) \mid 0 \leq i \leq m_{1}-1\right\} \cup\{(j, j, 0) \mid$ $k \leq j \leq 2 k\}$, an $\left(m_{1}+k+1\right)$-flower. Hence, the following result has been established. 
Lemma 4.10. Assume that $4 \leq k$ and $I S(k)=J S(k)$. Let $n=2 k+1$, then $(\{i \mid k+1 \leq$ $i \leq 2 k-1\} \cup\{2 k+1\}) \subset I S(n)$.

Combining Lemmas 4.6, 4.7, 4.8, 4.9 and 4.10 yields the following result.

Lemma 4.11. There exist pairs of latin squares of order $n$ yielding the following.

$$
I S(2)=\{0\} ; I S(3)=\{0,3\} ; \text { and } I S(n)=J S(n) \text { for } 4 \leq n .
$$

\subsection{Two disjoint $m$-flowers}

Let $2 \leq m$. In this section pairs of latin squares of order $n$, where $2 m \leq n$ that intersect precisely in two disjoint $m$-flowers and no other triples will be constructed. Note that in these constructions both $m$-flowers will be symbol- $m$-flowers.

Lemma 4.12. There exists a pair of latin squares of order five that intersect precisely in two disjoint 2-flowers.

Proof. The intersection (shown in bold) of the two latin squares shown below is the set of triples $A \cap B=\{(0,0,0),(1,1,0)\} \cup\{(2,2,1),(3,3,1)\}$, two disjoint 2-flowers.

$A:$\begin{tabular}{|c|c|c|c|c|}
\hline $\mathbf{0}$ & 1 & 2 & 3 & 4 \\
\hline 2 & $\mathbf{0}$ & 3 & 4 & 1 \\
\cline { 2 - 5 } 4 & 3 & $\mathbf{1}$ & 0 & 2 \\
\hline 3 & 2 & 4 & $\mathbf{1}$ & 0 \\
\hline 1 & 4 & 0 & 2 & 3 \\
\hline
\end{tabular}

$B:$\begin{tabular}{|c|c|c|c|c|}
\hline $\mathbf{0}$ & 3 & 4 & 2 & 1 \\
\hline 1 & $\mathbf{0}$ & 2 & 3 & 4 \\
\hline 3 & 2 & $\mathbf{1}$ & 4 & 0 \\
\hline 2 & 4 & 0 & $\mathbf{1}$ & 3 \\
\hline 4 & 1 & 3 & 0 & 2 \\
\hline
\end{tabular}

Once again, the construction splits into two cases.

\subsubsection{Case A: $n=2 k$}

First note the following exception, which follows from inspection of the latin squares of order four.

Lemma 4.13. There does not exist a pair of latin squares of order four that intersect precisely in two disjoint 2-flowers.

Two disjoint $m$-flowers: $m \in\{i \mid 0 \leq i \leq k-2\} \cup\{k\}$

A pair of latin squares, $\left\{L_{1}, L_{2}\right\}$, of order $2 k \geq 6$ that intersect precisely in two disjoint $m$-flowers, where $m \in\{i \mid 2 \leq i \leq k-2\} \cup\{k\}$, will now be constructed.

Consider the latin square $A=\{(0,0,0),(0,1,1),(1,0,1),(1,1,0)\}$ of order two. Let $m \in\{i \mid 2 \leq i \leq k-2\} \cup\{k\}$. Consider the pair of latin squares (constructed in Section 4.1), $\left\{U_{1}, U_{2}\right\}$, of order $k \geq 3$ whose intersection is the set of triples $\{(i, i, 0) \mid 0 \leq i \leq m\}$ (a symbol-m-flower). 
A pair of latin squares, $\left\{L_{1}, L_{2}\right\}$, of order $2 k$ that intersect precisely in two disjoint $m$-flowers will now be constructed. First, construct

$$
L_{1}=\{(0,0,0),(1,0,1),(0,1,1)\} \times U_{1} \cup\{(1,1,0)\} \times \sigma_{s}^{k} U_{1}
$$

Then, construct

$$
L_{2}=\{(0,0,0)\} \times U_{2} \cup\{(1,0,1),(0,1,1)\} \times \sigma_{s}^{k} U_{1} \cup\{(1,1,0)\} \times \sigma_{s}^{k} U_{2} .
$$

The intersection of $L_{1}$ and $L_{2}$ is the set of triples $\{(i, i, 0) \mid 0 \leq i \leq m-1\} \cup\{(j, j, 1) \mid$ $k \leq j \leq k+m-1\}$, two disjoint $m$-flowers. Hence, the following result has been established.

Lemma 4.14. Assume $6 \leq n=2 k$, then there exists a pair of latin squares of order $n$ that intersect precisely in two disjoint $m$-flowers, where $m \in\{i \mid 2 \leq i \leq k-2\} \cup\{k\}$.

Two disjoint $(k-1)$-flowers

A pair of latin squares, $\left\{L_{1}, L_{3}\right\}$, of order $2 k \geq 6$ that intersect precisely in two disjoint $(k-1)$-flowers will now be constructed.

Using the above, construct a pair of latin squares, $\left\{L_{1}, L_{2}\right\}$, that intersect precisely in two disjoint $k$-flowers. Then, let $L_{3}=\rho_{2 k-1}^{k-1} L_{2}$ (the map $\rho_{2 k-1}^{k-1}$ simply interchanges column $k-1$ with column $2 k-1$ in $L_{2}$ ).

This yields a pair of latin squares, of order $2 k$, whose intersection is precisely composed of two disjoint $(k-1)$-flowers. Hence, the following result has been established.

Lemma 4.15. Assume $3 \leq k$, then there exists a pair of latin squares of order $n=2 k$ that intersect in two disjoint $(k-1)$-flowers.

\subsubsection{Case B: $n=2 k+1$}

\section{Two disjoint $k$-flowers}

First, a pair of latin squares, $\left\{L_{1}^{\prime}, L_{2}\right\}$, of order $2 k+1 \geq 7$ that intersect precisely in one $(k+1)$-flower and one disjoint $k$-flower will be constructed. This pair is then used to construct a pair of latin squares, $\left\{L_{1}, L_{2}\right\}$, of order $2 k+1 \geq 7$ that intersect precisely in two disjoint $k$-flowers.

Consider a pair of latin squares (constructed in Section 4.1), $\left\{U_{1}, U_{2}\right\}$, of order $k \geq 3$, whose intersection is composed precisely of the triples $\{(i, i, 0) \mid 0 \leq i \leq k-1\}$ (a symbol $k$-flower). The symbols in $U_{1}$ and $U_{2}$ can be permuted, hence, without loss of generality $(k-2, k-1,1) \in U_{1}$.

First, a latin square $L_{1}^{\prime}$ will be constructed. From the previous section, the partial latin squares $C(2 k+1), D(2 k+1), F_{1}(2 k+1)$ and $G(2 k+1)$ will be made use of. In addition the following partial latin square will also be used.

$$
\begin{gathered}
H(2 k+1)=\{(i, j,(i-j-1(\bmod k+1))),(i,(i-1(\bmod k+1))+k, 0) \mid k \leq i, j \leq 2 k, i \neq j, \\
(i-1(\bmod k+1))+k \neq j,(i(\bmod k+1))+k \neq j\} .
\end{gathered}
$$




\section{Example 4.4.}

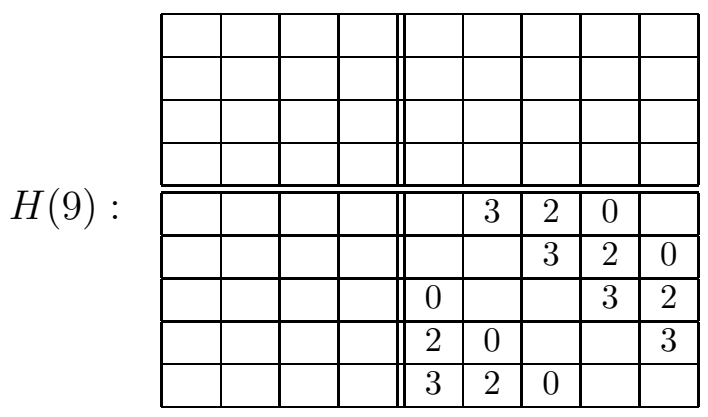

Construct the following latin square of order $2 k+1$;

$$
L_{1}^{\prime}=U_{1} \cup C(2 k+1) \cup D(2 k+1) \cup F_{1}(2 k+1) \cup H(2 k+1) \cup \sigma_{s}^{k+1} G(2 k+1) .
$$

The following set is claimed to be a latin square of order $2 k+1$;

$L_{2}=U_{2} \cup \sigma_{s}^{k, 2 k+1}\left(C(2 k+1) \cup D(2 k+1) \cup F_{1}(2 k+1)\right) \cup \psi_{k}^{2 k+1} H(2 k+1) \cup \sigma_{s}^{k+1} G(2 k+1)$.

Proof. The set of symbols in $H(2 k+1)$ is the set $\{h \mid 0 \leq h \leq k-1, h \neq 1\}$. Thus, $\psi_{k}^{2 k+1}$ merely permutes the symbols of $H$ such that there are no fixed points. Hence, as $L_{1}^{\prime}$ is a latin square of order $2 k+1$ it follows that $L_{2}$ is a latin square of order $2 k+1$.

The intersection of $L_{1}^{\prime}$ and $L_{2}$ is the set of triples $\{(i, i, 0) \mid 0 \leq i \leq k-1\} \cup\{(j, j, 1) \mid$ $k \leq j \leq 2 k\}$, a $k$-flower and a disjoint $k+1$-flower. Hence, the following result has been established.

Lemma 4.16. Assume $3 \leq k$. Let $n=2 k+1$, then there exists a pair of latin squares of order $n$ that intersect precisely in one $(k+1)$-flower and one disjoint $k$-flower.

Notice, $S_{1}=\{(k-2, k-1,1),(k-2,2 k,(3 k-1(\bmod k+1))+k),(2 k, k-1,(3 k-$ $1(\bmod k+1))+k),(2 k, 2 k, 1)\} \subset L_{1}^{\prime}$; forms one mate of the latin bitrade $\left(S_{1}, S_{2}\right)$ (an intercalate) where $S_{2}=\{(k-2, k-1,(3 k-1(\bmod k+1))+k),(k-2,2 k, 1),(2 k, k-$ $1,1),(2 k, 2 k,(3 k-1(\bmod k+1))+k)\}$. Hence, construct the latin square $L_{1}=\left(L_{1}^{\prime} \backslash\right.$ $\left.S_{1}\right) \cup S_{2}$.

The intersection of $L_{1}$ and $L_{2}$ is composed precisely of the triples in the set $\{(i, i, 0) \mid$ $0 \leq i \leq k-1\} \cup\{(j, j, 1) \mid k \leq j \leq 2 k-1\}$, two disjoint $k$-flowers. Thus, the following result has been established.

Lemma 4.17. Assume $3 \leq k$. Let $n=2 k+1$, then there exists a pair of latin squares of order $n$ that intersect precisely in two disjoint $k$-flowers.

Two disjoint $m$-flowers, where: $m \in\{i \mid 2 \leq i \leq k-1\}$

In the following, a pair of latin squares, $\left\{L_{3}, L_{2}\right\}$, that intersect precisely in two disjoint $m$-flowers, where $2 \leq m \leq k-1$, will be constructed.

Begin by using the above construction to form the pair, $\left\{L_{1}, L_{2}\right\}$, of latin squares of order $2 k+1$ that intersect in two disjoint $k$-flowers. 
Let $2 \leq m<k$. Note that if $L$ is a latin square of order $n$ and $0 \leq i, j \leq n$, then the map $\rho_{i}^{j}$ applied to a latin square $L$ simply swaps column $j$ with column $i$. Thus the following set is a latin square of order $2 k+1$;

$$
L_{3}=\rho_{1}^{k} \circ \rho_{2}^{k+1} \circ \rho_{3}^{k+2} \circ \ldots \circ \rho_{k-m}^{2 k-m-1} L_{1} .
$$

Furthermore, $L_{3}$ intersects $L_{2}$ precisely in two disjoint $m$-flowers, specifically $\{(0,0,0)$, $(i, i, 0) \mid k-m+1 \leq i \leq k-1\}$ and $\{(i, i, 1) \mid 2 k-m \leq i \leq 2 k-1\}$. Hence, the following result has been established.

Lemma 4.18. Assume $3 \leq k$. Let $n=2 k+1$, then there exists a pair of latin squares of order $n$ that intersect precisely in two disjoint $m$-flowers, where $m \in\{i \mid 2 \leq i \leq k-1\}$.

Combining Lemmas 4.14, 4.15, 4.17 and 4.18 yields the following result.

Lemma 4.19. There exists a pair of latin squares of order $n \geq 6$ whose intersection is composed of two disjoint $m$-flowers, where $2 \leq m \leq\left\lfloor\frac{n}{2}\right\rfloor$.

\section{3 $n=2 m+2 k+1$, where $0 \leq k \leq \frac{m-1}{2}$}

In this section pairs of latin squares of order $n=2 m+2 k+1$, where $k \in \mathbb{N}$ and $1 \leq$ $k \leq \frac{m-1}{2}$, that intersect precisely in three disjoint $m$-flowers, and pairs of latin squares of order $2 m+1$ that intersect precisely in three disjoint $m$-flowers and one other triple (by Lemma 4.2 there does not exist a pair of latin squares of order $2 m+1$ whose intersection is precisely three disjoint $m$-flowers) are constructed.

Example 4.5. A pair of latin squares, $\left\{L_{1}, L_{2}\right\}$, of order five that intersect in three disjoint 2-flowers and one other triple is shown below (the triples in the 2-flowers are shown in bold, while the additional triple is shown in italics).

$L_{1}:$\begin{tabular}{|c|c|c|c|c|}
\hline $\mathbf{4}$ & 3 & 1 & 0 & 2 \\
\hline 2 & $\mathbf{4}$ & 0 & 1 & 3 \\
\hline 0 & 2 & 3 & 4 & $\mathbf{1}$ \\
\hline 3 & 1 & 4 & 2 & $\mathbf{0}$ \\
\hline 1 & 0 & $\mathbf{2}$ & $\mathbf{3}$ & 4 \\
\hline
\end{tabular}

$L_{2}:$\begin{tabular}{|c|c|c|c|c|}
\hline $\mathbf{4}$ & 2 & 0 & 1 & 3 \\
\hline 1 & $\mathbf{4}$ & 3 & 0 & 2 \\
\cline { 2 - 5 } & 0 & 4 & 2 & $\mathbf{1}$ \\
\hline 2 & 3 & 1 & 4 & $\mathbf{0}$ \\
\hline 0 & 1 & $\mathbf{2}$ & $\mathbf{3}$ & 4 \\
\hline
\end{tabular}

Let $3 \leq m$.

First, latin squares, $L_{i}$, where $i \in\{1,2\}$, of order $2 m$ will be constructed. Then, using a method similar to prolongation pairs of latin squares of the required orders will be obtained.

Let $A=\{(0,0,0),(0,1,1),(1,0,1),(1,1,0)\}$.

Let $B$ be a latin square of order $m$ that contains at least $\left\lfloor\frac{m}{2}\right\rfloor+1$ transversals labelled $T^{i}$, where $0 \leq i \leq\left\lfloor\frac{m}{2}\right\rfloor$, (such a latin square exists by Lemma 3.2); without loss of generality, in the latin square $B, T^{0}=\{(i, i, i) \mid 0 \leq i \leq m-1\}$.

First a latin square, $L_{1}$, of order $2 m$ is constructed; $L_{1}=A \times B$.

Recall that $k \leq \frac{m-1}{2}$; construct the following latin square of order $2 m+2 k+1$;

$L_{1}(+2 k+1)=$ 


$$
\begin{aligned}
& L_{1} \backslash\left[\{(0,0,0)\} \times T^{0} \cup\{(1,0,1)\} \times T^{0} \cup\{(1,1,0)\} \times T^{0} \cup \cup_{1 \leq i \leq k}\left(A \times T^{i}\right)\right] \cup \\
& \gamma_{c}^{2 m}\left(\{(0,0,0)\} \times T^{0}\right) \cup \gamma_{s}^{2 m}\left(\{(0,0,0)\} \times T^{0}\right) \cup \gamma_{r}^{2 m}\left(\{(1,0,1)\} \times T^{0}\right) \cup \\
& \gamma_{c}^{2 m}\left(\{(1,0,1)\} \times T^{0}\right) \cup \gamma_{r}^{2 m}\left(\{(1,1,0)\} \times T^{0}\right) \cup \gamma_{s}^{2 m}\left(\{(1,1,0)\} \times T^{0}\right) \cup \\
& \{(m+i, i, i) \mid 0 \leq i \leq m-1\} \cup \\
& \bigcup_{1 \leq h \leq k} \\
& {\left[\gamma_{r}^{2 m+2 h-1}\left(\{(0,0,0)\} \times T^{h}\right) \cup \gamma_{c}^{2 m+2 h-1}\left(\{(0,0,0)\} \times T^{h}\right) \cup \gamma_{s}^{2 m+2 h-1}\left(\{(0,0,0)\} \times T^{h}\right) \cup\right.} \\
& \gamma_{r}^{2 m+2 h}\left(\left\{(1,0,1\} \times T^{h}\right) \cup \gamma_{c}^{2 m+2 h-1}\left(\{(1,0,1)\} \times T^{h}\right) \cup \gamma_{s}^{2 m+2 h}\left(\{(1,0,1)\} \times T^{h}\right) \cup\right. \\
& \gamma_{r}^{2 m+2 h}\left(\{(1,1,0)\} \times T^{h}\right) \cup \gamma_{c}^{2 m+2 h}\left(\{(1,1,0)\} \times T^{h}\right) \cup \gamma_{s}^{2 m+2 h-1}\left(\{(1,1,0)\} \times T^{h}\right) \cup \\
& \left.\gamma_{r}^{2 m+2 h-1}\left(\{(0,1,1)\} \times T^{h}\right) \cup \gamma_{c}^{2 m+2 h}\left(\{(0,1,1)\} \times T^{h}\right) \cup \gamma_{s}^{2 m+2 h}\left(\{(0,1,1)\} \times T^{h}\right)\right] \cup \\
& \{(2 m+j, 2 m+l, 2 m+(j+l(\bmod 2 k+1))) \mid 0 \leq j, l \leq 2 k\} .
\end{aligned}
$$

See Example A.2.

Next, a second latin square, $L_{2}$, of order $2 m$ is constructed; $L_{2}=A \times f_{\alpha} B$, where $f_{(0,0,0)}=f_{(0,1,1)}=\phi_{s}^{m}, f_{(1,0,1)}=\sigma_{c}^{m}$, and $f_{(1,1,0)}=\sigma_{r}^{m}$.

Construct the following latin square of order $2 m+2 k+1$;

$L_{2}(+2 k+1)=$

$L_{2} \backslash\left[\{(0,0,0)\} \times \phi_{s}^{m} T^{0} \cup\{(1,0,1)\} \times \sigma_{c}^{m} T^{0} \cup\{(1,1,0)\} \times \sigma_{r}^{m} T^{0}\right) \cup \bigcup_{1 \leq i \leq k}(A \times$ $\left.\left.f_{\alpha} T^{i}\right)\right] \cup$

$$
\begin{aligned}
& \gamma_{c}^{2 m}\left(\{(0,0,0)\} \times \phi_{s}^{m} T^{0}\right) \cup \gamma_{s}^{2 m}\left(\{(0,0,0)\} \times \phi_{s}^{m} T^{0}\right) \cup \gamma_{r}^{2 m}\left(\{(1,0,1)\} \times \sigma_{c}^{m} T^{0}\right) \cup \\
& \gamma_{c}^{2 m}\left(\{(1,0,1)\} \times \sigma_{c}^{m} T^{0}\right) \cup \gamma_{r}^{2 m}\left(\{(1,1,0)\} \times \sigma_{r}^{m} T^{0}\right) \cup \gamma_{s}^{2 m}\left(\{(1,1,0)\} \times \sigma_{r}^{m} T^{0}\right) \cup \\
& \{(m+i, i+1(\bmod m), i-1(\bmod m)) \mid 0 \leq i \leq m-1\} \cup \\
& \bigcup_{1 \leq h \leq k} \\
& {\left[\gamma_{r}^{2 m+2 h}\left(\{(0,0,0)\} \times \phi_{s}^{m} T^{h}\right) \cup \gamma_{c}^{2 m+2 h}\left(\{(0,0,0)\} \times \phi_{s}^{m} T^{h}\right) \cup \gamma_{s}^{2 m+2 h}(\{(0,0,0)\} \times\right.} \\
& \left.\phi_{s}^{m} T^{h}\right) \cup \\
& \left.\gamma_{r}^{2 m+2 h-1}(\{1,0,1)\} \times \sigma_{c}^{m} T^{h}\right) \cup \gamma_{c}^{2 m+2 h}\left(\{(1,0,1)\} \times \sigma_{c}^{m} T^{h}\right) \cup \gamma_{s}^{2 m+2 h-1}(\{(1,0,1)\} \times \\
& \left.\sigma_{c}^{m} T^{h}\right) \cup \\
& \gamma_{r}^{2 m+2 h-1}\left(\{(1,1,0)\} \times \sigma_{r}^{m} T^{h}\right) \cup \gamma_{c}^{2 m+2 h-1}\left(\{(1,1,0)\} \times \sigma_{r}^{m} T^{h}\right) \cup \gamma_{s}^{2 m+2 h}(\{(1,1,0)\} \times \\
& \left.\sigma_{r}^{m} T^{h}\right) \cup \\
& \gamma_{r}^{2 m+2 h}\left(\{(0,1,1)\} \times \phi_{s}^{m} T^{h}\right) \cup \gamma_{c}^{2 m+2 h-1}\left(\{(0,1,1)\} \times \phi_{s}^{m} T^{h}\right) \cup \gamma_{s}^{2 m+2 h-1}(\{(0,1,1)\} \times \\
& \left.\left.\phi_{s}^{m} T^{h}\right)\right] \cup \\
& \{(2 m+j, 2 m+l, 2 m+(j+l+1(\bmod 2 k+1))) \mid 0 \leq j, l \leq 2 k\} .
\end{aligned}
$$

See Example A.3.

Consider the intersection of $L_{1}(+2 k+1)$ and $L_{2}(+2 k+1)$. Note that 
for $k \geq 1$ this intersection is composed precisely of the disjoint $m$-flowers $\{(i, i, 2 m) \mid$ $0 \leq i \leq m-1\},\{(m+i, 2 m, m+i) \mid 0 \leq i \leq m-1\}$ and $\{(2 m, m+i, i) \mid 0 \leq i \leq$ $m-1\}$

for $k=0$ this intersection is composed precisely of the disjoint $m$-flowers $\{(i, i, 2 m) \mid$ $0 \leq i \leq m-1\},\{(m+i, 2 m, m+i) \mid 0 \leq i \leq m-1\}$ and $\{(2 m, m+i, i) \mid 0 \leq i \leq$ $m-1\}$ and the additional triple $(2 m, 2 m, 2 m)$.

See Example A.4.

The existence of latin squares of order seven that intersect precisely in three disjoint 2-flowers is shown in [13]. Also, Example 4.5 shows a pair of latin squares of order five that intersect precisely in three disjoint 2-flowers and one other triple. Hence, the following result has been established.

Lemma 4.20. Assume $2 \leq m$ and $n=2 m+2 k+1$, where $1 \leq k \leq \frac{m-1}{2}$, then there exists a pair of latin squares of order $n$ that intersect precisely in three disjoint $m$-flowers. Furthermore, there exists a pair of latin squares of order $2 m+1$ that intersect precisely in three disjoint $m$-flowers and one other triple.

\section{4 $n=2 m+2 k$, where $1 \leq k \leq \frac{m}{2}$}

Example 4.6. A pair of latin squares, $\left\{L_{1}, L_{2}\right\}$, of order six that intersect in three disjoint 2 -flowers is shown below (the triples in the 2-flowers are shown in bold).

$L_{1}:$\begin{tabular}{|c|c|c|c|c|c|}
\hline $\mathbf{0}$ & 2 & 4 & 3 & 5 & 1 \\
\hline 1 & $\mathbf{0}$ & 5 & 4 & 2 & 3 \\
\hline 3 & 5 & $\mathbf{1}$ & $\mathbf{2}$ & 0 & 4 \\
\hline 2 & 4 & 0 & 1 & $\mathbf{3}$ & 5 \\
\hline 5 & 1 & 3 & 0 & $\mathbf{4}$ & 2 \\
\hline 4 & 3 & 2 & 5 & 1 & 0 \\
\hline
\end{tabular}

$L_{2}:$\begin{tabular}{|c|c|c|c|c|c|}
\hline $\mathbf{0}$ & 1 & 5 & 4 & 2 & 3 \\
\hline 5 & $\mathbf{0}$ & 2 & 3 & 1 & 4 \\
\hline 4 & 3 & $\mathbf{1}$ & $\mathbf{2}$ & 5 & 0 \\
\hline 1 & 5 & 4 & 0 & $\mathbf{3}$ & 2 \\
\hline 3 & 2 & 0 & 5 & $\mathbf{4}$ & 1 \\
\hline 2 & 4 & 3 & 1 & 0 & 5 \\
\hline
\end{tabular}

Let $3 \leq m$. In the remainder of this section pairs of latin squares of order $n=2 m+2 k$, where $3 \leq m, k \in \mathbb{N} \cup\{0\}$ and $1 \leq k \leq \frac{m}{2}$, that intersect precisely in three disjoint $m$ flowers will be constructed.

The constructions all start by forming a latin square, $L_{i}$, where $i \in\{1,2\}$, of order $2 m$ and then increasing this order through a method similar to prolongation.

For the first latin square (of the pair) two separate constructions are used, one for when $k=1$ and one for when $2 \leq k \leq \frac{m}{2}$. The same construction, for both $k=1$ and $2 \leq k \leq \frac{m}{2}$, is used for the second latin square.

Let $A=\{(0,0,0),(0,1,1),(1,0,1),(1,1,0)\}$.

Let $B$ be a latin square of order $m$ that contains at least $\left\lfloor\frac{m}{2}\right\rfloor+1$ transversals labelled $T^{i}$, where $0 \leq i \leq\left\lfloor\frac{m}{2}\right\rfloor$ (such a latin square exists by Lemma 3.2); without loss of generality, in the latin square $B, T^{0}=\{(i, i, i) \mid 0 \leq i \leq m-1\}$.

First a latin square, $L_{1}$, of order $2 m$ is constructed; $L_{1}=A \times B$.

Now construct the following latin square of order $2 m+2$;

$L_{1}^{\prime}(+2)=$ 


$$
\begin{aligned}
& L_{1} \backslash\left[A \times T^{0}\right] \cup \\
& \gamma_{c}^{2 m}\left(\{ ( 0 , 0 , 0 ) \times T ^ { 0 } ) \cup \gamma _ { s } ^ { 2 m } \left(\{ ( 0 , 0 , 0 ) \times T ^ { 0 } ) \cup \gamma _ { r } ^ { 2 m } \left(\left\{(0,0,0) \times T^{0}\right) \cup\right.\right.\right. \\
& \gamma_{c}^{2 m}\left(\{ ( 1 , 0 , 1 ) \times T ^ { 0 } ) \cup \gamma _ { s } ^ { 2 m + 1 } \left(\{ ( 1 , 0 , 1 ) \times T ^ { 0 } ) \cup \gamma _ { r } ^ { 2 m + 1 } \left(\left\{(1,0,1) \times T^{0}\right) \cup\right.\right.\right. \\
& \gamma_{c}^{2 m+1}\left(\{ ( 0 , 1 , 1 ) \times T ^ { 0 } ) \cup \gamma _ { s } ^ { 2 m + 1 } \left(\{ ( 0 , 1 , 1 ) \times T ^ { 0 } ) \cup \gamma _ { r } ^ { 2 m } \left(\left\{(0,1,1) \times T^{0}\right) \cup\right.\right.\right. \\
& \gamma_{c}^{2 m+1}\left(\{ ( 1 , 1 , 0 ) \times T ^ { 0 } ) \cup \gamma _ { s } ^ { 2 m } \left(\{ ( 1 , 1 , 0 ) \times T ^ { 0 } ) \cup \gamma _ { r } ^ { 2 m + 1 } \left(\left\{(1,1,0) \times T^{0}\right) \cup\right.\right.\right. \\
& \{(2 m+j, 2 m+l, 2 m+(j+l(\bmod 2))) \mid 0 \leq j, l \leq 1\} .
\end{aligned}
$$

See Example A.5.

Notice, $\left(R_{1}=\{(2 m-1, i, x),(2 m+1, i, m+i) \mid 0 \leq i \leq m-2,(2 m-1, i, x) \in\right.$ $\left.\left.L_{1}^{\prime}(+2)\right\}, R_{2}=\left\{(2 m-1, i, m+i),(2 m+1, i, x) \mid 0 \leq i \leq m-2,(2 m-1, i, x) \in L_{1}^{\prime}(+2)\right\}\right)$ is a latin bitrade where $R_{1} \subset L_{1}^{\prime}(+2)$.

Similarly, $\left(S_{1}=\{(2 m-1, m-1,2 m+1),(2 m-1,2 m, 2 m-1),(2 m+1, m-1,2 m-\right.$ $1),(2 m+1,2 m, 2 m+1)\}, S_{2}=\{(2 m-1, m-1,2 m-1),(2 m-1,2 m, 2 m+1),(2 m+$ $1, m-1,2 m+1),(2 m+1,2 m, 2 m-1)\})$ is a latin bitrade where $S_{1} \subset L_{1}^{\prime}(+2)$.

Hence, construct the latin square $L_{1}(+2)=\left(L_{1}^{\prime}(+2) \backslash\left(R_{1} \cup S_{1}\right)\right) \cup\left(R_{2} \cup S_{2}\right)$. See Example A.6

Let $2 \leq k \leq \frac{m}{2}$; consider the latin square of order $2 m+2 k ; L_{1}(+2 k)=$

$$
\begin{aligned}
& L_{1} \backslash\left[\bigcup_{0 \leq h \leq k-1} A \times T^{h}\right] \\
& \bigcup_{0 \leq h \leq 1} \\
& {\left[\gamma_{r}^{2 m+h}\left(\left\{(0,0,0) \times T^{h}\right\}\right) \cup \gamma_{c}^{2 m+2 h}\left(\{(0,0,0)\} \times T^{h}\right) \cup \gamma_{s}^{2 m+2 h}\left(\left\{(0,0,0) \times T^{h}\right\}\right) \cup\right.} \\
& \gamma_{r}^{2 m+h}\left(\left\{(0,1,1) \times T^{h}\right\}\right) \cup \gamma_{c}^{2 m+2 h+1}\left(\{(0,1,1)\} \times T^{h}\right) \cup \gamma_{s}^{2 m+2 h+1}\left(\left\{(0,1,1) \times T^{h}\right\}\right) \cup \\
& \gamma_{r}^{2 m+2+h}\left(\left\{(1,0,1) \times T^{h}\right\}\right) \cup \gamma_{c}^{2 m+2 h}\left(\{(1,0,1)\} \times T^{h}\right) \cup \gamma_{s}^{2 m+2 h+1}\left(\left\{(1,0,1) \times T^{h}\right\}\right) \cup \\
& \left.\gamma_{r}^{2 m+3-h}\left(\left\{(1,1,0) \times T^{h}\right\}\right) \cup \gamma_{c}^{2 m+2 h+1}\left(\{(1,1,0)\} \times T^{h}\right) \cup \gamma_{s}^{2 m+2 h}\left(\left\{(1,1,0) \times T^{h}\right\}\right)\right] \cup \\
& \bigcup_{2 \leq h \leq k-1} \\
& {\left[\gamma_{r}^{2 m+2 h}\left(\left\{(0,0,0) \times T^{h}\right\}\right) \cup \gamma_{c}^{2 m+2 h}\left(\{(0,0,0)\} \times T^{h}\right) \cup \gamma_{s}^{2 m+2 h}\left(\left\{(0,0,0) \times T^{h}\right\}\right) \cup\right.} \\
& \gamma_{r}^{2 m+2 h}\left(\left\{(0,1,1) \times T^{h}\right\}\right) \cup \gamma_{c}^{2 m+2 h+1}\left(\{(0,1,1)\} \times T^{h}\right) \cup \gamma_{s}^{2 m+2 h+1}\left(\left\{(0,1,1) \times T^{h}\right\}\right) \cup \\
& \gamma_{r}^{2 m+2 h+1}\left(\left\{(1,0,1) \times T^{h}\right\}\right) \cup \gamma_{c}^{2 m+2 h}\left(\{(1,0,1)\} \times T^{h}\right) \cup \gamma_{s}^{2 m+2 h+1}\left(\left\{(1,0,1) \times T^{h}\right\}\right) \cup \\
& \left.\gamma_{r}^{2 m+2 h+1}\left(\left\{(1,1,0) \times T^{h}\right\}\right) \cup \gamma_{c}^{2 m+2 h+1}\left(\{(1,1,0)\} \times T^{h}\right) \cup \gamma_{s}^{2 m+2 h}\left(\left\{(1,1,0) \times T^{h}\right\}\right)\right] \cup \\
& \{(2 m+j, 2 m+l, 2 m+(j+l(\bmod 2 k))) \mid 0 \leq j, l \leq 2 k-1\} .
\end{aligned}
$$

See Example A.7.

Next, a latin square, $L_{2}$, of order $2 m$ will be constructed; $L_{2}=A \times f_{\alpha} B$, where $f_{(0,0,0)}=\sigma_{s}^{m}, f_{(1,0,1)}=f_{(0,1,1)}=\sigma_{r}^{m}$ and $f_{(1,1,0)}=\sigma_{c}^{m}$.

Let $1 \leq k \leq \frac{m}{2}$. Consider the latin square of order $2 m+2 k$;

$L_{2}(+2 k)=$ 


$$
\begin{aligned}
& L_{2} \backslash\left[\bigcup_{0 \leq h \leq k-1} A \times\left\{f_{\alpha} T^{h}\right\}_{\alpha \in A}\right] \cup \\
& \gamma_{r}^{2 m}\left(\{(0,0,0)\} \times \sigma_{s}^{m} T^{0}\right) \cup \gamma_{c}^{2 m}\left(\{(0,0,0)\} \times \sigma_{s}^{m} T^{0}\right) \cup \gamma_{s}^{2 m}\left(\{(0,0,0)\} \times \sigma_{s}^{m} T^{0}\right) \cup \\
& \gamma_{r}^{2 m}\left(\{(0,1,1)\} \times \sigma_{r}^{m} T^{0}\right) \cup \gamma_{c}^{2 m+1}\left(\{(0,1,1)\} \times \sigma_{r}^{m} T^{0}\right) \cup \gamma_{s}^{2 m+1}\left(\{(0,1,1)\} \times \sigma_{r}^{m} T^{0}\right) \cup \\
& \gamma_{r}^{2 m+1}\left(\{(1,0,1)\} \times \sigma_{r}^{m} T^{0}\right) \cup \gamma_{c}^{2 m}\left(\{(1,0,1)\} \times \sigma_{r}^{m} T^{0}\right) \cup \gamma_{s}^{2 m+1}\left(\{(1,0,1)\} \times \sigma_{r}^{m} T^{0}\right) \cup \\
& \gamma_{r}^{2 m+1}\left(\{(1,1,0)\} \times \sigma_{c}^{m} T^{0}\right) \cup \gamma_{c}^{2 m+1}\left(\{(1,1,0)\} \times \sigma_{c}^{m} T^{0}\right) \cup \gamma_{s}^{2 m}\left(\{(1,1,0)\} \times \sigma_{c}^{m} T^{0}\right) \\
& \bigcup_{1 \leq h \leq k-1} \\
& {\left[\gamma_{r}^{2 m+2 h+1}\left(\{(0,0,0)\} \times \sigma_{s}^{m} T^{h}\right) \cup \gamma_{c}^{2 m+2 h+1}\left(\{(0,0,0)\} \times \sigma_{s}^{m} T^{h}\right) \cup \gamma_{s}^{2 m+2 h+1}(\{(0,0,0)\} \times\right.} \\
& \left.\sigma_{s}^{m} T^{h}\right) \cup \\
& \gamma_{r}^{2 m+2 h+1}\left(\{(0,1,1)\} \times \sigma_{r}^{m} T^{h}\right) \cup \gamma_{c}^{2 m+2 h}\left(\{(0,1,1)\} \times \sigma_{r}^{m} T^{h}\right) \cup \gamma_{s}^{2 m+2 h}(\{(0,1,1)\} \times \\
& \left.\sigma_{r}^{m} T^{h}\right) \cup \\
& \gamma_{r}^{2 m+2 h}\left(\{(1,0,1)\} \times \sigma_{r}^{m} T^{h}\right) \cup \gamma_{c}^{2 m+2 h+1}\left(\{(1,0,1)\} \times \sigma_{r}^{m} T^{h}\right) \cup \gamma_{s}^{2 m+2 h}(\{(1,0,1)\} \times \\
& \left.\sigma_{r}^{m} T^{h}\right) \cup \\
& \gamma_{r}^{2 m+2 h}\left(\{(1,1,0)\} \times \sigma_{c}^{m} T^{h}\right) \cup \gamma_{c}^{2 m+2 h}\left(\{(1,1,0)\} \times \sigma_{c}^{m} T^{h}\right) \cup \gamma_{s}^{2 m+2 h+1}(\{(1,1,0)\} \times \\
& \left.\left.\sigma_{c}^{m} T^{h}\right)\right] \cup \\
& \{(2 m+j, 2 m+l, 2 m+(j+l+1(\bmod 2 k))) \mid 0 \leq j, l \leq 2 k-1\} .
\end{aligned}
$$

See Example A.8

The intersection of $L_{1}(+2)$ and $L_{2}(+2)$ is composed precisely of the disjoint $m$-flowers $\{(i, i, 2 m) \mid 0 \leq i \leq m-1\},\{(2 m, m+i, m+i) \mid 0 \leq i \leq m-1\}$ and $\{(m+i, 2 m+1, i) \mid$ $0 \leq i \leq m-1\}$. See Example A.9.

Recall that, Example 4.6 shows a pair of latin squares of order six that intersect precisely in three disjoint 2-flowers. Thus, the following result has been established.

Lemma 4.21. Assume $2 \leq m$ then there exists a pair of latin squares of order $2 m+2$ that intersect precisely in three disjoint $m$-flowers.

Let $4 \leq m$. Let $2 \leq k \leq \frac{m}{2}$. Consider the intersection of $L_{1}(+2 k)$ and $L_{2}(+2 k)$. This intersection is composed precisely of the disjoint $m$-flowers $\{(i, i, 2 m) \mid 0 \leq i \leq m-1\}$, $\{(2 m, m+i, m+i) \mid 0 \leq i \leq m-1\}$ and $\{(m+i, 2 m+1, i) \mid 0 \leq i \leq m-1\}$. See Example A.10. Hence, the following result has been established.

Lemma 4.22. Assume $4 \leq m$ and $2 \leq k \leq \frac{m-1}{2}$ then there exists a pair of latin squares of order $2 m+2 k$ that intersect precisely in three disjoint $m$-flowers.

Combining Lemmas 4.21 and 4.22 yields the following result.

Lemma 4.23. Assume $2 \leq m$ and $1 \leq k \leq \frac{m-1}{2}$ then there exists a pair of latin squares of order $2 m+2 k$ that intersect precisely in three disjoint $m$-flowers. 


\section{5 $3 m+1 \leq n \leq 4 m$}

Let $3 \leq m$. In this section pairs of latin squares of order $n=3 m+k$, where $k \in \mathbb{N}$ and $1 \leq k \leq m$, that intersect precisely in three or four disjoint $m$-flowers will be constructed.

First, a pair of latin squares, of order $3 m+k$, that intersect in three disjoint $m$-flowers is constructed. Then this construction is modified to obtain a pair of latin squares, of order $3 m+k$ that intersect in four disjoint $m$-flowers.

Let $A=\{(0,0,0),(0,1,2),(0,2,1),(1,0,2),(1,1,1),(1,2,0),(2,0,1),(2,1,0),(2,2,2)\}$.

Let $B$ be a latin square of order $m$ that contains at least $\left\lfloor\frac{m}{2}\right\rfloor+1$ transversals labelled $T^{i}$, where $0 \leq i \leq\left\lfloor\frac{m}{2}\right\rfloor$ (such a latin square exists by Lemma 3.2); without loss of generality, in the latin square $B, T^{0}=\{(i, i, i) \mid 0 \leq i \leq m-1\}$.

Begin by constructing a latin square, $L_{1}$, of order $3 m ; L_{1}=A \times B$.

Let $k_{1}=\left\lceil\frac{k-1}{2}\right\rceil$ and $k_{2}=\left\lfloor\frac{k-1}{2}\right\rfloor$. Also let, $1 \leq i \leq k_{1}$ and $1 \leq j \leq k_{2}$. By Lemma 3.3 the sets $\{(t, t, t) \mid 0 \leq t \leq 2\} \times T^{0},\{(0,1,2),(1,2,0),(2,0,1)\} \times T^{i}$ and $\{(t, t, t) \mid 0 \leq t \leq$ $2\} \times T^{j}$ are transversals in $L_{1}$. So, $k$-prolongate down the following $k$ transversals in $L_{1}$ :

$$
\begin{aligned}
& T_{0}=\{(t, t, t) \mid 0 \leq t \leq 2\} \times T^{0} ; \\
& T_{i}=\{(0,1,2),(1,2,0),(2,0,1)\} \times T^{i}, \text { where } 1 \leq i \leq k_{1} ; \text { and } \\
& T_{k_{1}+j}=\{(t, t, t) \mid 0 \leq t \leq 2\} \times T^{j}, \text { where } 1 \leq j \leq k_{2} ;
\end{aligned}
$$

using the completing square $\{(3 m+h, 3 m+l, 3 m+(h+l(\bmod \mathrm{k}))) \mid 0 \leq h, l \leq k-1\}$ to form the latin square $L_{1}(+k)$. See Example A.11.

Now, a latin square, $L_{2}$, of order $3 m$ will be constructed. Let $A^{\prime}$ be the set $A \backslash$ $\{(1,1,1),(2,2,2)\}$. Then, $L_{2}=A \times\left\{f_{\alpha} B\right\}_{\alpha \in A}$, where $f_{\alpha}=\sigma_{s}^{m}$ when $\alpha \in A^{\prime}, f_{(1,1,1)}=\sigma_{c}^{m}$, and $f_{(2,2,2)}=\sigma_{r}^{m}$.

As before, let $k_{1}=\left\lceil\frac{k-1}{2}\right\rceil$ and $k_{2}=\left\lfloor\frac{k-1}{2}\right\rfloor$. Also, as before, let $1 \leq i \leq k_{1}$ and $1 \leq j \leq$ $k_{2}$. By Lemma 3.3 the sets $\{(t, t, t) \mid 0 \leq t \leq 2\} \times f_{\alpha} T^{0},\{(t, t, t) \mid 0 \leq t \leq 2\} \times f_{\alpha} T^{i}$ and $\{(0,1,2),(1,2,0),(2,0,1)\} \times f_{\alpha} T^{j}$ are transversals in $L_{2}$. So, $k$-prolongate down the following $k$ transversals

$$
\begin{aligned}
& T_{i}=\{(h, h, h) \mid 0 \leq h \leq 2\} \times f_{\alpha} T^{i}, \text { where } 0 \leq i \leq k_{1} ; \text { and } \\
& T_{k_{1}+j}=\{(0,1,2),(1,2,0),(2,0,1)\} \times f_{\alpha} T^{j}, \text { where } 1 \leq j \leq k_{2}
\end{aligned}
$$

and use the completing square $\{(3 m+h, 3 m+l, 3 m+(h+l+1(\bmod k))) \mid 0 \leq h, l \leq k-1\}$ to form the latin square $L_{2}(+k)$. See Example A.12.

The latin squares $L_{1}(+k)$ and $L_{2}(+k)$ intersect precisely in three disjoint $m$-flowers if $2 \leq k$ (namely $\{(i, i, i) \mid 0 \leq i \leq m-1\},\{(m+i, 3 m, m+i) \mid 0 \leq i \leq m-1\}$ and $\{(3 m, 2 m+i, 2 m+i) \mid 0 \leq i \leq m-1\}$ (see Example A.13)) and precisely in the same three disjoint $m$-flowers and one other triple (namely $(3 m, 3 m, 3 m)$ ) if $k=1$.

Notice,

$$
\begin{gathered}
R_{1}=\{(0, m, 2 m),(0,2 m, m),(0,3 m, 0),(m, 0,2 m),(m, m, 3 m),(m, 2 m, 0), \\
(2 m, 0, m),(2 m, 2 m, 3 m),(2 m, 3 m, 2 m),(3 m, 0,0),(3 m, m, m),(3 m, 3 m, 3 m)\} \subset L_{1}(+1) ;
\end{gathered}
$$


this set is one mate of the latin bitrade $\left(R_{1}, R_{2}\right)$, where

$$
\begin{gathered}
R_{2}=\{(0, m, m),(0,2 m, 0),(0,3 m, 2 m),(m, 0,0),(m, m, 2 m),(m, 2 m, 3 m),(2 m, 0,2 m) \\
,(2 m, 2 m, m),(2 m, 3 m, 3 m),(3 m, 0, m),(3 m, m, 3 m),(3 m, 3 m, 0)\} .
\end{gathered}
$$

Construct the latin square $L_{3}(+1)=\left(L_{1} \backslash R_{1}\right) \cup R_{2}$. See Example A.14.

The latin squares $L_{3}(+1)$ and $L_{2}(+1)$ intersect precisely in three disjoint $m$-flowers. Hence, the following result has been established.

Lemma 4.24. Assume $3 \leq m$ and $3 m+1 \leq n \leq 4 m$, then there exists a pair of latin squares of order $n$ that intersect precisely in three disjoint $m$-flowers.

Next a latin square $L_{4}(+k)$, where $1 \leq k \leq m$, is constructed in the same manner as $L_{2}(+k)$ except letting $f_{(2,1,0)}=v_{s}^{m}$.

The latin squares $L_{1}(+k)$ and $L_{4}(+k)$, where $2 \leq k \leq m$, and $L_{3}(+1)$ and $L_{4}(+1)$ intersect precisely in four disjoint $m$-flowers, namely $\{(i, i, i) \mid 0 \leq i \leq m-1\},\{(m+$ $i, 3 m, m+i) \mid 0 \leq i \leq m-1\},\{(3 m, 2 m+i, 2 m+i) \mid 0 \leq i \leq m-1\}$ and $\{(2 m+i, m+$ $i, m-1) \mid(i, j, m-1) \in B\}$. Thus, the following result has been proved.

Lemma 4.25. Assume $3 \leq m$ and $3 m+1 \leq n \leq 4 m$, then there exists a pair of latin squares of order $n$ that intersect precisely in four disjoint $m$-flowers.

\section{$4.6 n=4 m+1$}

In this section pairs of latin squares of order $n=4 m+1$ that intersect precisely in 3,4 and 5 disjoint $m$-flowers, are constructed.

Let $A=\{(0,0,0),(0,1,3),(0,2,1),(0,3,2),(1,0,2),(1,1,1),(1,2,3),(1,3,0),(2,0,3)$, $(2,1,0),(2,2,2),(2,3,1),(3,0,1),(3,1,2),(3,2,0),(3,3,3)\}$.

Let $B$ be a latin square of order $m$ that contains a transversal, labelled $T^{0}$ (such a latin square exists by Lemma 3.2); without loss of generality, in the latin square $B$, $T^{0}=\{(i, i, i) \mid 0 \leq i \leq m-1\}$.

First the latin square, $L_{1}$, of order $4 m$ is constructed; $L_{1}=A \times B$. By Lemma 3.3, the set $\{(i, i, i) \mid 0 \leq i \leq 3\} \times T^{0}$ is a transversal in $L_{1}$. Thus, prolongate down this transversal to form the latin square $L_{1}(+1)$ of order $4 m+1$. See Example A.15.

Now a latin square, $L_{2}$, of order $4 m$ is constructed. Let $A^{\prime}=A \backslash\{(1,1,1),(2,2,2),(3,3$, $3)\}$. Then, $L_{2}=A \times\left\{f_{\alpha} B\right\}_{\alpha \in A}$, where $f_{\alpha}=\sigma_{s}^{m}$ when $\alpha \in A^{\prime}, f_{(1,1,1)}=\sigma_{r}^{m}, f_{(2,2,2)}=\sigma_{c}^{m}$ and $f_{(3,3,3)}=i d$.

By Lemma 3.3 the set $\{(i, i, i) \mid 0 \leq i \leq 3\} \times f_{\alpha} T^{0}$ is a transversal in $L_{2}$. Prolongate down this transversal to form the latin square $L_{2}^{\prime}(+1)$.

Note that the triples in the set $R_{1}=\left\{(x, y, z) \mid 3 m \leq x, y \leq 4 m,(x, y, z) \in L_{1}(+1)\right\}$ occur in both $L_{1}(+1)$ and $L_{2}^{\prime}(+1)$. Also, note that these triples form one mate of a latin bitrade $\left(R_{1}, R_{2}\right)$, where $R_{2}=\left\{(x, y,(z-3 m+1(\bmod m+1))+3 m) \mid(x, y, z) \in R_{1}\right\}$ $\left(R_{1}\right.$ is in fact a latin square where $\left.N=\{i \mid 3 m \leq i \leq 4 m\}\right)$. Construct $L_{2}(+1)=$ $\left(L_{2}^{\prime}(+1) \backslash R_{1}\right) \cup R_{2}$. See Example A.16. 
The latin squares $L_{1}(+1)$ and $L_{2}(+1)$ intersect precisely in three disjoint $m$-flowers, namely $\{(i, i, i) \mid 0 \leq i \leq m-1\},\{(4 m, m+i, m+i) \mid 0 \leq i \leq m-1\}$ and $\{(2 m+$ $i, 4 m, 2 m+i) \mid 0 \leq i \leq m-1\}$. Hence, the following result has been proved.

Lemma 4.26. Assume $3 \leq m$ then there exists a pair of latin squares of order $4 m+1$ that intersect precisely in three disjoint $m$-flowers.

Now construct a latin square $L_{3}(+1)$ in the same manner as $L_{2}(+1)$ except let $f_{(3,2,0)}=$ $v_{s}^{m}$; and construct $L_{4}(+1)$ in the same manner as $L_{2}(+1)$ except let $f_{(3,2,0)}=v_{s}^{m}$ and $f_{(1,3,0)}=\epsilon_{s}^{m}$.

The latin squares $L_{1}(+1)$ and $L_{3}(+1)$ intersect precisely in four disjoint $m$-flowers (namely $\{(i, i, i) \mid 0 \leq i \leq m-1\},\{(4 m, m+i, m+i) \mid 0 \leq i \leq m-1\},\{(2 m+i, 4 m, 2 m+$ $i) \mid 0 \leq i \leq m-1\}$ and $\{(3 m+i, 2 m+j, m-1) \mid(i, j, m-1) \in B\})$ and the latin squares $L_{1}(+1)$ and $L_{4}(+1)$ intersect precisely in five disjoint $m$-flowers (namely $\{(i, i, i) \mid 0 \leq i \leq$ $m-1\},\{(4 m, m+i, m+i) \mid 0 \leq i \leq m-1\},\{(2 m+i, 4 m, 2 m+i) \mid 0 \leq i \leq m-1\}$, $\{(3 m+i, 2 m+j, m-1) \mid(i, j, m-1) \in B\}$ and $\{(m+i, 3 m+j, m-2) \mid(i, j, m-2) \in B\})$. Hence, the following result has been established.

Lemma 4.27. Assume $3 \leq m$. Then there exists a pair of latin squares of order $4 m+1$ that intersect precisely in four disjoint $m$-flowers and there exists a pair of latin squares of order $4 m+1$ that intersect precisely in five disjoint $m$-flowers.

\section{7 $4 m+2 \leq n \leq 6 m+2$}

Let $3 \leq m$. In this section pairs of latin squares of order $n$ are constructed that intersect in: $3,4,5$ or 6 disjoint $m$-flowers when $4 m+2 \leq n \leq 5 m+1$; in 3, 4, 5, 6 or 7 disjoint $m$-flowers when $5 m+2 \leq n \leq 6 m+1$; and in $3,4,5,6,7$ or 8 disjoint $m$-flowers when $n=6 m+2$.

First a pair of latin squares of order $n$, where $4 m+2 \leq n \leq 6 m+2$, that intersect in 5 disjoint $m$-flowers will be constructed.

Let $B$ be a latin square of order $m$ that contains at least $\left\lceil\frac{m}{2}\right\rceil+1$ transversals labelled $T^{i}$, where $0 \leq i \leq\left\lceil\frac{m}{2}\right\rceil$ (such a latin square exists by Lemma 3.2); without loss of generality, in the latin square $B, T^{0}=\{(i, i, i) \mid 0 \leq i \leq m-1\}$.

Let $A_{1}=\{(0,0,0),(0,1,1),(0,2,2),(0,3,3),(1,0,1),(1,1,0),(1,2,3),(1,3,2),(2,0,2)$, $(2,1,3),(2,2,0),(2,3,1),(3,0,3),(3,1,2),(3,2,1),(3,3,0)\}$. Notice that $A_{1}=U_{1} \cup U_{2} \cup$ $U_{3} \cup U_{4}$, where:

$$
\begin{aligned}
& U_{1}=\{(0,0,0),(1,2,3),(2,3,1),(3,1,2)\} ; U_{2}=\{(0,1,1),(1,3,2),(2,2,0),(3,0,3)\} \\
& U_{3}=\{(0,2,2),(1,0,1),(2,1,3),(3,3,0)\} ; U_{4}=\{(0,3,3),(1,1,0),(2,0,2),(3,2,1)\} .
\end{aligned}
$$

Also, note that each $U_{i}$, where $1 \leq i \leq 4$, is a transversal in $A_{1}$ and that these transversals are disjoint.

$$
A_{1}: \begin{array}{|c|c|c|c|}
\hline 0 & 1 & 2 & 3 \\
\hline 1 & 0 & 3 & 2 \\
\hline 2 & 3 & 0 & 1 \\
\hline 3 & 2 & 1 & 0 \\
\hline
\end{array}
$$

Transversals:

\begin{tabular}{|c|c|c|c|}
\hline$U_{1}$ & $U_{2}$ & $U_{3}$ & $U_{4}$ \\
\hline$U_{3}$ & $U_{4}$ & $U_{1}$ & $U_{2}$ \\
\hline$U_{4}$ & $U_{3}$ & $U_{2}$ & $U_{1}$ \\
\hline$U_{2}$ & $U_{1}$ & $U_{4}$ & $U_{3}$ \\
\hline
\end{tabular}


First, a latin square, $L_{1}$, of order $4 m$ is constructed; $L_{1}=A_{1} \times B$.

Next, let $2 \leq k \leq 2 m+2$ and $k-2=k_{1}+k_{2}+k_{3}+k_{4}$, where $0 \leq k_{4} \leq k_{3} \leq k_{2} \leq$ $k_{1} \leq\left\lceil\frac{m}{2}\right\rceil$. Construct the following latin square of order $4 m+k$;

$$
\begin{aligned}
& L_{1}^{\prime}(+k)= \\
& L_{1} \backslash\left[\left(\left(A_{1} \backslash\{(0,1,1),(0,3,3),(2,1,3),(2,3,1)\}\right) \times T^{0}\right) \cup \bigcup_{1 \leq h_{1} \leq k_{1}}\left(U_{1} \times T^{h_{1}}\right) \cup\right. \\
& \left.\bigcup_{1 \leq h_{2} \leq k_{2}}\left(U_{2} \times T^{h_{2}}\right) \cup \bigcup_{1 \leq h_{3} \leq k_{3}}\left(U_{3} \times T^{h_{3}}\right) \cup \bigcup_{1 \leq h_{4} \leq k_{4}}\left(U_{4} \times T^{h_{4}}\right)\right] \cup \\
& \gamma_{r}^{4 m}\left(\{(1,0,1),(1,1,0),(1,2,3),(1,3,2)\} \times T^{0}\right) \cup \\
& \gamma_{r}^{4 m+1}\left(\{(3,0,3),(3,1,2),(3,2,1),(3,3,0)\} \times T^{0}\right) \cup \\
& \gamma_{c}^{4 m}\left(\{(3,0,3),(2,0,2),(1,0,1),(0,0,0)\} \times T^{0}\right) \cup \\
& \gamma_{c}^{4 m+1}\left(\{(3,2,1),(2,2,0),(1,2,3),(0,2,2)\} \times T^{0}\right) \cup \\
& \gamma_{s}^{4 m}\left(\{(0,0,0),(1,1,0),(2,2,0),(3,3,0)\} \times T^{0}\right) \cup \\
& \gamma_{s}^{4 m+1}\left(\{(0,2,2),(1,3,2),(2,0,2),(3,1,2)\} \times T^{0}\right) \cup \\
& \{(m+i, i, i),(m+i, 2 m+i, 2 m+i),(3 m+i, i, 2 m+i),(3 m+i, 2 m+i, i) \mid 0 \leq i \leq \\
& m-1\} \cup \\
& \bigcup_{1 \leq h_{1} \leq k_{1}}\left(\gamma_{r}^{4 m+1+h_{1}}\left(U_{1} \times T^{h_{1}}\right) \cup \gamma_{c}^{4 m+1+h_{1}}\left(U_{1} \times T^{h_{1}}\right) \cup \gamma_{s}^{4 m+1+h_{1}}\left(U_{1} \times T^{h_{1}}\right)\right) \cup \\
& \bigcup_{1 \leq h_{2} \leq k_{2}}\left(\gamma_{r}^{4 m+1+k_{1}+h_{2}}\left(U_{2} \times T^{h_{2}}\right) \cup \gamma_{c}^{4 m+1+k_{1}+h_{2}}\left(U_{2} \times T^{h_{2}}\right) \cup \gamma_{s}^{4 m+1+k_{1}+h_{2}}\left(U_{2} \times T^{h_{2}}\right)\right) \cup \\
& \bigcup_{1 \leq h_{3} \leq k_{3}}\left(\gamma _ { r } ^ { 4 m + 1 + k _ { 1 } + k _ { 2 } + h _ { 3 } } ( U _ { 3 } \times T ^ { h _ { 3 } } ) \cup \gamma _ { c } ^ { 4 m + 1 + k _ { 1 } + k _ { 2 } + h _ { 3 } } ( U _ { 3 } \times T ^ { h _ { 3 } } ) \cup \gamma _ { s } ^ { 4 m + 1 + k _ { 1 } + k _ { 2 } + h _ { 3 } } \left(U_{3} \times\right.\right. \\
& \left.\left.T^{h_{3}}\right)\right) \cup \\
& \bigcup_{1 \leq h_{4} \leq k_{4}}\left(\gamma_{r}^{4 m+1+k_{1}+k_{2}+k_{3}+h_{4}}\left(U_{4} \times T_{4}^{h}\right) \quad \cup \quad \gamma_{c}^{4 m+1+k_{1}+k_{2}+k_{3}+h_{4}}\left(U_{4} \times T^{h_{4}}\right) \quad \cup\right. \\
& \left.\gamma_{s}^{4 m+1+k_{1}+k_{2}+k_{3}+h_{4}}\left(U_{4} \times T_{4}^{h}\right)\right) \cup \\
& \{(4 m+j, 4 m+l, 4 m+(j+l(\bmod k))) \mid 0 \leq j, l \leq k-1\} .
\end{aligned}
$$

See Example A.17.

Notice, $R_{1}=\{(m+i, m+i, 4 m),(m+i, 3 m+i, 4 m+1),(3 m+i, m+i, 4 m+1),(3 m+$ $i, 3 m+i, 4 m) \mid 0 \leq i \leq m-1\} \subset L_{1}^{\prime}(+k)$; this set is one mate of the latin bitrade $\left(R_{1}, R_{2}\right)$, where $R_{2}=\{(m+i, m+i, 4 m+1),(m+i, 3 m+i, 4 m),(3 m+i, m+i, 4 m),(3 m+i, 3 m+$ $i, 4 m+1) \mid 0 \leq i \leq m-1\}$.

Hence, construct the latin square $L_{1}(+k)=\left(L_{1}^{\prime}(+k) \backslash R_{1}\right) \cup R_{2}$.

Let $A_{2}=\{(0,0,0),(0,1,1),(0,2,3),(0,3,2),(1,0,1),(1,1,0),(1,2,2),(1,3,3),(2,0,3)$, $(2,1,2),(2,2,0),(2,3,1),(3,0,2),(3,1,3),(3,2,1),(3,3,0)\}$. Notice that $A_{2}=U_{1}^{\prime} \cup U_{2}^{\prime} \cup$ $U_{3}^{\prime} \cup U_{4}^{\prime}$, where:

$$
\begin{aligned}
& U_{1}^{\prime}=\{(0,3,2),(1,1,0),(2,0,3),(3,2,1)\} ; U_{2}^{\prime}=\{(0,2,3),(1,0,1),(2,1,2),(3,3,0)\} \\
& U_{3}^{\prime}=\{(0,1,1),(1,3,3),(2,2,0),(3,0,2)\} ; U_{4}^{\prime}=\{(0,0,0),(1,2,2),(2,3,1),(3,1,3)\} .
\end{aligned}
$$

Also, note that each $U_{i}^{\prime}$, where $1 \leq i \leq 4$, is a transversal in $A_{2}$ and that these transversals are disjoint. 


$A_{2}:$\begin{tabular}{|c|c|c|c|}
\hline 0 & 1 & 3 & 2 \\
\hline 1 & 0 & 2 & 3 \\
\hline 3 & 2 & 0 & 1 \\
\hline 2 & 3 & 1 & 0 \\
\hline
\end{tabular}$\quad$ Transversals: \begin{tabular}{|c|c|c|c|c|}
\hline$U_{4}^{\prime}$ & $U_{3}^{\prime}$ & $U_{2}^{\prime}$ & $U_{1}^{\prime}$ \\
\hline$U_{2}^{\prime}$ & $U_{1}^{\prime}$ & $U_{4}^{\prime}$ & $U_{3}^{\prime}$ \\
\hline$U_{1}^{\prime}$ & $U_{2}^{\prime}$ & $U_{3}^{\prime}$ & $U_{4}^{\prime}$ \\
\hline$U_{3}^{\prime}$ & $U_{4}^{\prime}$ & $U_{1}^{\prime}$ & $U_{2}^{\prime}$ \\
\hline
\end{tabular}

Now, the latin square, $L_{2}$, of order $4 m$ will be constructed. $L_{2}=A_{2} \times f_{\alpha} B$, where $f_{(0,3,2)}=f_{(1,2,2)}=f_{(1,3,3)}=f_{(2,0,3)}=f_{(3,1,3)}=i d, f_{(2,1,2)}=f_{(3,0,2)}=f_{(0,0,0)}=f_{(0,1,1)}=$ $f_{(1,0,1)}=f_{(1,1,0)}=f_{(2,3,1)}=f_{(0,2,3)}=\sigma_{s}^{m}, f_{(3,3,0)}=\phi_{s}^{m}, f_{(2,2,0)}=\sigma_{c}^{m}$ and $f_{(3,2,1)}=\sigma_{r}^{m}$.

\begin{tabular}{|c|c|c|c|}
\hline$\sigma_{s}^{m}$ & $\sigma_{s}^{m}$ & $\sigma_{s}^{m}$ & $i d$ \\
\hline$\sigma_{s}^{m}$ & $\sigma_{s}^{m}$ & $i d$ & $i d$ \\
\hline$i d$ & $\sigma_{s}^{m}$ & $\sigma_{c}^{m}$ & $\sigma_{s}^{m}$ \\
\hline$\sigma_{s}^{m}$ & $i d$ & $\sigma_{r}^{m}$ & $\phi_{s}^{m}$ \\
\hline
\end{tabular}

Let $2 \leq k \leq 2 m+2$, and $k_{i}$, where $1 \leq i \leq 4$, be the same as in the construction of $L_{1}^{\prime}(+k)$. So consider the following latin square of order $4 m+k$.

$L_{2}(+k)=$

$$
\begin{aligned}
& L_{2} \backslash\left[\left((A \backslash\{(0,1,1),(1,3,3),(2,0,3),(2,3,1)\}) \times f_{\alpha} T^{0}\right) \cup \bigcup_{1 \leq h_{1} \leq k_{1}}\left(U_{1}^{\prime} \times f_{\alpha} T^{h_{1}}\right) \cup\right. \\
& \left.\bigcup_{1 \leq h_{2} \leq k_{2}}\left(U_{2}^{\prime} \times f_{\alpha} T^{h_{2}}\right) \cup \bigcup_{1 \leq h_{3} \leq k_{3}}\left(U_{3}^{\prime} \times f_{\alpha} T^{h_{3}}\right) \cup \bigcup_{1 \leq h_{4} \leq k_{4}}\left(U_{4}^{\prime} \times f_{\alpha} T^{h_{4}}\right)\right] \cup \\
& \gamma_{r}^{4 m}\left(\{(1,0,1),(1,1,0),(0,2,3),(0,3,2)\} \times f_{\alpha} T^{0}\right) \cup \\
& \gamma_{r}^{4 m+1}\left(\{(3,0,2),(3,1,3),(3,2,1),(3,3,0)\} \times f_{\alpha} T^{0}\right) \cup \\
& \gamma_{c}^{4 m}\left(\{(0,0,0),(1,0,1),(2,1,2),(3,1,3)\} \times f_{\alpha} T^{0}\right) \cup \\
& \gamma_{c}^{4 m+1}\left(\{(0,2,3),(1,2,2),(2,2,0),(3,2,1)\} \times f_{\alpha} T^{0}\right) \cup \\
& \gamma_{s}^{4 m}\left(\{(0,0,0),(1,1,0),(2,2,0),(3,3,0)\} \times f_{\alpha} T^{0}\right) \cup \\
& \gamma_{s}^{4 m+1}\left(\{(0,3,2),(1,2,2),(2,1,2),(3,0,2)\} \times f_{\alpha} T^{0}\right) \cup \\
& \{(i, 2 m+i, 2 m+i),(m+i, i,(i+1(\bmod m))),(3 m+i, m+i, 2 m+(i+1(\bmod m))), \\
& (3 m+(i+1(\bmod m)), 2 m+i,(i-1(\bmod m))) \mid 0 \leq i \leq m-1\} \cup \\
& \bigcup_{1 \leq h_{1} \leq k_{1}}\left(\gamma_{r}^{4 m+1+h_{1}}\left(U_{1}^{\prime} \times f_{\alpha} T^{h_{1}}\right) \cup \gamma_{c}^{4 m+1+h_{1}}\left(U_{1}^{\prime} \times f_{\alpha} T^{h_{1}}\right) \cup \gamma_{s}^{4 m+1+h_{1}}\left(U_{1}^{\prime} \times f_{\alpha} T^{h_{1}}\right)\right) \cup \\
& \bigcup_{1 \leq h_{2} \leq k_{2}}\left(\gamma _ { r } ^ { 4 m + 1 + k _ { 1 } + h _ { 2 } } ( U _ { 2 } ^ { \prime } \times f _ { \alpha } T ^ { h _ { 2 } } ) \cup \gamma _ { c } ^ { 4 m + 1 + k _ { 1 } + h _ { 2 } } ( U _ { 2 } ^ { \prime } \times f _ { \alpha } T ^ { h _ { 2 } } ) \cup \gamma _ { s } ^ { 4 m + 1 + k _ { 1 } + h _ { 2 } } \left(U_{2}^{\prime} \times\right.\right. \\
& \left.\left.f_{\alpha} T^{h_{2}}\right)\right) \cup \quad \\
& \bigcup_{1 \leq h_{3} \leq k_{3}}\left(\gamma_{r}^{4 m+1+k_{1}+k_{2}+h_{3}}\left(U_{3}^{\prime} \times f_{\alpha} T^{h_{3}}\right) \quad \cup \quad \gamma_{c}^{4 m+1+k_{1}+k_{2}+h_{3}}\left(U_{3}^{\prime} \times f_{\alpha} T^{h_{3}}\right)\right. \\
& \left.\gamma_{s}^{4 m+1+k_{1}+k_{2}+h_{3}}\left(U_{3}^{\prime} \times f_{\alpha} T^{h_{3}}\right)\right) \cup \quad \cup \\
& \bigcup_{1 \leq h_{4} \leq k_{4}}\left(\gamma_{r}^{4 m+1+k_{1}+k_{2}+k_{3}+h_{4}}\left(U_{4}^{\prime} \times f_{\alpha} T^{h_{4}}\right) \quad \cup \quad \gamma_{c}^{4 m+1+k_{1}+k_{2}+k_{3}+h_{4}}\left(U_{4}^{\prime} \times f_{\alpha} T^{h_{4}}\right)\right. \\
& \left.\gamma_{s}^{4 m+1+k_{1}+k_{2}+k_{3}+h_{4}}\left(U_{4}^{\prime} \times f_{\alpha} T^{h_{4}}\right)\right) \cup \\
& \{(4 m+j, 4 m+l, 4 m+(j+l+1(\bmod k))) \mid 0 \leq j, l \leq k-1\} .
\end{aligned}
$$

See Example A.18.

The latin squares $L_{1}(+k)$ and $L_{2}(+k)$ intersect precisely in five disjoint $m$-flowers (namely $\{(i, i, 4 m) \mid 0 \leq i \leq m-1\},\{(2 m+i, 4 m+1, i) \mid 0 \leq i \leq m-1\},\{3 m+i, 4 m, 3 m+$ $i \mid 0 \leq i \leq m-1\},\{(4 m, 3 m+i, 2 m+i) \mid 0 \leq i \leq m-1\}$ and $\{(4 m+1,2 m+i, m+i) \mid$ $0 \leq i \leq m-1\}$ ) and no other triples. See Example A.19.

Thus, the following result has been established. 
Lemma 4.28. Assume $3 \leq m$ and $4 m+2 \leq n \leq 6 m+2$, then there exists a pair of latin squares of order $n$ that intersect precisely in five disjoint $m$-flowers.

Notice $S_{1}=\{(m+i, m+i, 4 m),(m+i, 2 m+i, 4 m+1),(2 m+i, m+i, 4 m+1),(2 m+$ $i, 2 m+(i+1(\bmod m)), 4 m) \mid 0 \leq i \leq m-1\} \subset L_{2}(+k)$; this set of triples is one mate of the latin bitrade $\left(S_{1}, S_{2}\right)$, where $S_{2}=\{(m+i, m+i, 4 m+1),(m+i, 2 m+i, 4 m),(2 m+$ $i, m+i, 4 m),(2 m+i, 2 m+(i+1(\bmod m)), 4 m+1) \mid 0 \leq i \leq m-1\}$.

Construct the latin square $L_{3}(+k)=\left(L_{2}(+k) \backslash S_{1}\right) \cup S_{2}$.

The latin squares $L_{1}(+k)$ and $L_{3}(+k)$ intersect in precisely in six disjoint $m$-flowers, specifically $\{(i, i, 4 m) \mid 0 \leq i \leq m-1\},\{(2 m+i, 4 m+1, i) \mid 0 \leq i \leq m-1\},\{3 m+$ $i, 4 m, 3 m+i \mid 0 \leq i \leq m-1\},\{(4 m, 3 m+i, 2 m+i) \mid 0 \leq i \leq m-1\},\{(4 m+1,2 m+$ $i, m+i) \mid 0 \leq i \leq m-1\}$ and $\{(m+i, m+i, 4 m+1) \mid 0 \leq i \leq m-1\}$. Hence, the following result has been established.

Lemma 4.29. Assume $3 \leq m$ and $4 m+2 \leq n \leq 6 m+2$, then there exists a pair of latin squares of order $n$ that intersect precisely in six disjoint $m$-flowers.

Let $m+2 \leq k \leq 2 m+2$; let $V_{1}=\left\{(i, j, u) \mid 4 m \leq i, j \leq 4 m+k-1,(i, j, u) \in L_{1}(+k)\right\}$ and $W_{1}=\left\{(i, j, v) \mid 4 m \leq i, j \leq 4 m+k-1,(i, j, v) \in L_{3}(+k)\right\}$.

From Lemma 4.11 there exists a pair of latin squares of order $k$, where $m+2 \leq k \leq$ $2 m+2$, that intersect precisely in one symbol- $m$-flower. By relabelling the rows, columns and symbols (the same permutations are applied to both squares) the symbol- $m$-flower is the set $\{(i, i, 2) \mid 2 \leq i \leq m+1\}$. Denote this pair of latin squares by $\left\{V_{2}^{\prime}, W_{2}^{\prime}\right\}$.

Let $V_{2}=\delta^{4 m} V_{2}^{\prime}$ and $W_{2}=\delta^{4 m} W_{2}^{\prime}$. Note that, $\left(V_{1}, V_{2}\right)$ and $\left(W_{1}, W_{2}\right)$ are latin biswaps. So, construct the latin squares $L_{4}(+k)=\left(L_{1}(+k) \backslash V_{1}\right) \cup V_{2}$ and $L_{5}(+k)=\left(L_{1}(+k) \backslash\right.$ $\left.W_{1}\right) \cup W_{2}$.

Thus, the latin squares $L_{4}(+k)$ and $L_{5}(+k)$ intersect precisely in seven disjoint $m$ flowers, specifically $\{(i, i, 4 m) \mid 0 \leq i \leq m-1\},\{(2 m+i, 4 m+1, i) \mid 0 \leq i \leq m-1\}$, $\{3 m+i, 4 m, 3 m+i \mid 0 \leq i \leq m-1\},\{(4 m, 3 m+i, 2 m+i) \mid 0 \leq i \leq m-1\}$, $\{(4 m+1,2 m+i, m+i) \mid 0 \leq i \leq m-1\},\{(m+i, m+i, 4 m+1) \mid 0 \leq i \leq m-1\}$ and $\{(4 m+2+i, 4 m+2+i, 4 m+2) \mid 0 \leq i \leq m-1\}$. Hence, the following result has been established.

Lemma 4.30. Assume $3 \leq m$ and $5 m+2 \leq n \leq 6 m+2$, then there exists a pair of latin squares of order $n$ that intersect precisely in seven disjoint $m$-flowers.

Let $k=2 m+2$; let $X_{1}=\left\{(i, j, u) \mid 4 m \leq i, j \leq 4 m+k-1,(i, j, u) \in L_{1}(+k)\right\}$ and $Y_{1}=\left\{(i, j, v) \mid 4 m \leq i, j \leq 4 m+k-1,(i, j, v) \in L_{3}(+k)\right\}$.

From Lemma 4.19 there exists a pair of latin squares of order $k=2 m+2$ that intersect precisely in two disjoint symbol- $m$-flowers. By relabelling the rows, columns and symbols (the same permutations are applied to both squares) the symbol-m-flowers are the sets $\{(i, i, 2) \mid 2 \leq i \leq m+1\}$ and $\{(i, i, 3) \mid m+2 \leq i \leq 2 m+1\}$. Denote this pair of latin squares by $\left\{X_{2}^{\prime}, Y_{2}^{\prime}\right\}$.

Let $X_{2}=\delta^{4 m} X_{2}^{\prime}$ and $Y_{2}=\delta^{4 m} Y_{2}^{\prime}$. Note that, $\left(X_{1}, X_{2}\right)$ and $\left(Y_{1}, Y_{2}\right)$ are latin biswaps. Construct the latin squares $L_{6}(+k)=\left(L_{1}(+k) \backslash X_{1}\right) \cup X_{2}$ and $L_{7}(+k)=\left(L_{3}(+k) \backslash Y_{1}\right) \cup Y_{2}$. 
The latin squares $L_{6}(+k)$ and $L_{7}(+k)$ intersect precisely in eight disjoint $m$-flowers, specifically $\{(i, i, 4 m) \mid 0 \leq i \leq m-1\},\{(2 m+i, 4 m+1, i) \mid 0 \leq i \leq m-1\},\{3 m+$ $i, 4 m, 3 m+i \mid 0 \leq i \leq m-1\},\{(4 m, 3 m+i, 2 m+i) \mid 0 \leq i \leq m-1\},\{(4 m+1,2 m+i, m+$ i) $\mid 0 \leq i \leq m-1\},\{(m+i, m+i, 4 m+1) \mid 0 \leq i \leq m-1\},\{(4 m+2+i, 4 m+2+i, 4 m+2) \mid$ $0 \leq i \leq m-1\}$ and $\{(5 m+2+i, 5 m+2+i, 4 m+3) \mid 0 \leq i \leq m-1\}$. Hence, the following result has been established.

Lemma 4.31. Assume $3 \leq m$, then there exists a pair of latin squares of order $6 m+2$ that intersect precisely in eight disjoint $m$-flowers.

Now a pair of latin squares of order $n$, where $4 m+2 \leq n \leq 6 m+2$, that intersect in 3 disjoint $m$-flowers; and a pair of latin squares of order $n$, where $4 m+2 \leq n \leq 6 m+2$, that intersect in 4 disjoint $m$-flowers will be constructed.

Let $B$ be a latin square of order $m$ that contains at least $\left\lceil\frac{m}{2}\right\rceil+1$ transversals labelled $T^{i}$, where $0 \leq i \leq\left\lceil\frac{m}{2}\right\rceil$ (such a latin square exists by Lemma 3.2); without loss of generality, in the latin square $B, T^{0}=\{(i, i, i) \mid 0 \leq i \leq m-1\}$.

Let $A=\{(0,0,0),(0,1,1),(0,2,2),(0,3,3),(1,0,1),(1,1,0),(1,2,3),(1,3,2),(2,0,2)$, $(2,1,3),(2,2,0),(2,3,1),(3,0,3),(3,1,2),(3,2,1),(3,3,0)\}$. Notice that $A=U_{1} \cup U_{2} \cup$ $U_{3} \cup U_{4}$, where:

$$
\begin{aligned}
& U_{1}=\{(0,0,0),(1,2,3),(2,3,1),(3,1,2)\} ; U_{2}=\{(0,1,1),(1,3,2),(2,2,0),(3,0,3)\} \\
& U_{3}=\{(0,2,2),(1,0,1),(2,1,3),(3,3,0)\} ; U_{4}=\{(0,3,3),(1,1,0),(2,0,2),(3,2,1)\}
\end{aligned}
$$

Also note that each $U_{i}$, where $1 \leq i \leq 4$, is a transversal in $A$ and that these transversals are disjoint.

$A:$\begin{tabular}{|c|c|c|c|}
\hline 0 & 1 & 2 & 3 \\
\hline 1 & 0 & 3 & 2 \\
\hline 2 & 3 & 0 & 1 \\
\hline 3 & 2 & 1 & 0 \\
\hline
\end{tabular}$\quad$ Transversals: \begin{tabular}{|c|c|c|c|c|}
\hline$U_{1}$ & $U_{2}$ & $U_{3}$ & $U_{4}$ \\
\hline$U_{3}$ & $U_{4}$ & $U_{1}$ & $U_{2}$ \\
\hline$U_{4}$ & $U_{3}$ & $U_{2}$ & $U_{1}$ \\
\hline$U_{2}$ & $U_{1}$ & $U_{4}$ & $U_{3}$ \\
\hline
\end{tabular}

First, a latin square, $N_{1}$, of order $4 m$ is constructed; $N_{1}=A \times B$.

Let $2 \leq k \leq 2 m+2$ and $k-2=k_{1}+k_{2}+k_{3}+k_{4}$, where $0 \leq k_{4} \leq k_{3} \leq k_{2} \leq k_{1} \leq\left\lceil\frac{m}{2}\right\rceil$. Let $0 \leq j \leq\left\lceil\frac{m}{2}\right\rceil$ and $1 \leq i \leq 4$; by Lemma 3.3 the sets $U_{i} \times T^{j}$ are transversals in $N_{1}$. Consider the transversals

$$
\begin{aligned}
& D_{0}=U_{1} \times T^{0} ; D_{1}=U_{3} \times T^{0} ; D_{1+h_{1}}=U_{1} \times T^{h_{1}}, \text { where } 1 \leq h_{1} \leq k_{1} ; \\
& D_{k_{1}+1+h_{2}}=U_{2} \times T^{h_{2}}, \text { where } 1 \leq h_{2} \leq k_{2} ; D_{k_{1}+k_{2}+1+h_{3}}=U_{1} \times T^{h_{3}}, \text { where } 1 \leq h_{3} \leq k_{3} ;
\end{aligned}
$$$$
\text { and } D_{k_{1}+k_{2}+k_{3}+1+h_{4}}=U_{1} \times T^{h_{4}} \text {, where } 1 \leq h_{4} \leq k_{4}
$$

Hence, $k$-prolongate down the $k$ disjoint transversals $D_{i}$, where $0 \leq i \leq k-1$, and use the completing square $\{(4 m+i, 4 m+j, 4 m+(i+j(\bmod k))) \mid 0 \leq i, j \leq k-1\}$ to form the latin square $N_{1}(+k)$. See Example A.20.

Next, the latin square, $N_{2}$, of order $4 m$ will be constructed; $N_{2}=A \times f_{\alpha} B$, where $f_{(0,0,0)}=f_{(0,1,1)}=f_{(0,2,2)}=f_{(0,3,3)}=f_{(1,1,0)}=f_{(1,3,2)}=f_{(2,0,2)}=f_{(2,1,3)}=f_{(2,2,0)}=$ $f_{(2,3,1)}=f_{(3,0,3)}=f_{(3,2,1)}=\sigma_{s}^{m}, f_{(1,0,1)}=f_{(3,1,2)}=\sigma_{c}^{m}$ and $f_{(1,2,3)}=f_{(3,3,0)}=\sigma_{r}^{m}$. 


\begin{tabular}{|c|c|c|c|}
\hline$\sigma_{s}^{m}$ & $\sigma_{s}^{m}$ & $\sigma_{s}^{m}$ & $\sigma_{s}^{m}$ \\
\hline$\sigma_{c}^{m}$ & $\sigma_{s}^{m}$ & $\sigma_{r}^{m}$ & $\sigma_{s}^{m}$ \\
\hline$\sigma_{s}^{m}$ & $\sigma_{s}^{m}$ & $\sigma_{s}^{m}$ & $\sigma_{s}^{m}$ \\
\hline$\sigma_{s}^{m}$ & $\sigma_{c}^{m}$ & $\sigma_{s}^{m}$ & $\sigma_{r}^{m}$ \\
\hline
\end{tabular}

By Lemma 3.3 the sets $\left\{U_{i} \times f_{\alpha} T^{j}\right\}$ are transversals in $N_{2}$. Consider the transversals $E_{0}=U_{3} \times f_{\alpha} T^{0} ; E_{1}=U_{1} \times f_{\alpha} T^{0} ; E_{1+h_{1}}=U_{2} \times f_{\alpha} T^{h_{1}}$, where $1 \leq h_{1} \leq k_{1} ;$ $E_{k_{1}+1+h_{2}}=U_{3} \times f_{\alpha} T^{h_{2}}$, where $1 \leq h_{2} \leq k_{2} ; E_{k_{1}+k_{2}+1+h_{3}}=U_{4} \times f_{\alpha} T^{h_{3}}$, where $1 \leq h_{3} \leq k_{3}$; and $E_{k_{1}+k_{2}+k_{3}+1+h_{4}}=U_{1} \times f_{\alpha} T^{h_{4}}$, where $1 \leq h_{4} \leq k_{4}$.

Let $\zeta_{r}=\zeta_{v}=\left(\begin{array}{ll}1 & 2\end{array}\right)$. Now, $\left(\zeta_{r}, \zeta_{c}\right)$ - $k$-prolongate down the $k$ disjoint transversals $E_{i}$, where $0 \leq i \leq k-1$, and use the completing square $\{(4 m+i, 4 m+j, 4 m+(i+j+$ $1(\bmod k))) \mid 0 \leq i, j \leq k-1\}$ to form the latin square $N_{2}(+k)$. See Example A.21.

The latin squares $N_{1}(+k)$ and $N_{2}(+k)$ intersect precisely in four disjoint $m$-flowers, namely $\{(4 m, 2 m+i, 3 m+i) \mid 0 \leq i \leq m-1\},\{(4 m+1,3 m+i, i) \mid 0 \leq i \leq m-1\}$, $\{(3 m+i, 4 m, 2 m+i) \mid 0 \leq i \leq m-1\}$ and $\{(m+i, 4 m+1, m+i) \mid 0 \leq i \leq m-1\}$. See Example A.22.

Hence, the following result has been proved.

Lemma 4.32. Assume $3 \leq m$ and $4 m+2 \leq n \leq 6 m+2$, then there exists a pair of latin squares of order $n$ that intersect precisely in four disjoint $m$-flowers.

Now a latin square $N_{3}(+k)$ is constructed. This construction is the same as the construction for $N_{2}(+k)$ except that $f_{(1,2,3)}=\sigma_{s}^{m}$.

The latin squares $N_{1}(+k)$ and $N_{3}(+k)$ intersect precisely in three disjoint $m$-flowers, namely $\{(4 m+1,3 m+i, i) \mid 0 \leq i \leq m-1\},\{(3 m+i, 4 m, 2 m+i) \mid 0 \leq i \leq m-1\}$ and $\{(m+i, 4 m+1, m+i) \mid 0 \leq i \leq m-1\}$. Hence, the following result has been established.

Lemma 4.33. Assume $3 \leq m$ and $4 m+2 \leq n \leq 6 m+2$, then there exists a pair of latin squares of order $n$ that intersect precisely in three disjoint $m$-flowers.

\section{$4.86 m+3 \leq n$}

In this section constructions for the remaining cases required to prove Theorem 1 will be provided.

Let $2 \leq m$.

Let $A$ be a latin square of order $l \geq 3$ that contains at least three disjoint transversals labelled $U_{0}, U_{1}$ and $U_{2}$; without loss of generality, in the latin square $A, U_{0}=\{(i, i, i) \mid$ $0 \leq i \leq l-1\}$.

Let $B_{1}$ be a latin square of order $2 m+1$ which contains $2 m+1$ disjoint transversals. Without loss of generality $(0,0,0) \in B_{1}$. Label the $2 m+1$ disjoint transversals in $B_{1}$ as $V_{1}^{j}$, where $0 \leq j \leq 2 m$, such that $(0,0,0) \in V_{1}^{0}$. Let $B_{2}=\sigma_{s}^{2 m+1} B_{1}$ and label the transversal $\sigma_{s}^{2 m+1} V_{1}^{j}$ as $V_{2}^{j}$, where $0 \leq j \leq 2 m$. Note that $B_{1} \cap B_{2}=\emptyset$.

The existence of the above latin squares $\left(A\right.$ and $\left.B_{1}\right)$ is guaranteed by Lemma 3.2. 
Let $C_{1}$ and $C_{2}$ be a pair of latin squares of order $2 m+1$ that intersect in three disjoint $m$-flowers and one other triple, namely the triple $(0,0,0)$ (note that this triple will not be disjoint from the $m$-flowers). Such a pair exists by Lemma 4.20.

Let $D_{1}$ and $D_{2}$ be a pair of latin squares of order $2 m+1$ that intersect precisely in two disjoint $m$-flowers. Without loss of generality $(0,0,0) \in D_{1}$ and $(0,0,0) \notin D_{2}$. Such a pair exists by Lemma 4.19 .

Let $E_{1}$ and $E_{2}$ be a pair of latin squares of order $2 m+1$ that intersect precisely in one $m$-flower. Without loss of generality $(0,0,0) \in E_{1}$ and $(0,0,0) \notin E_{2}$. Such a pair exists by Lemma 4.11 .

Recall $3 \leq l$. A pair of latin squares, $\left\{L_{1}^{h}(+k), L_{2}^{h}(+k)\right\}$, of order $l(2 m+1)+k$, where $0 \leq k \leq 2 m$, that intersect in precisely $h$, where $0 \leq h \leq 3 l$, disjoint $m$-flowers will now be constructed.

Determine $b, c, d, e \in \mathbb{N} \cup\{0\}$ such that $3 c+2 d+e=h$ and $b+c+d+e=l$ (it should be noted that this is always possible).

First, construct the latin square $L_{1}^{h}(+0)^{\prime}$ of order $l(2 m+1)$.

$$
L_{1}^{h}(+0)^{\prime}=\{(i, i, i) \mid 0 \leq i \leq c-1\} \times C_{1} \cup\{(i, i, i) \mid c \leq i \leq c+d-1\} \times D_{1} \cup
$$
$\{(i, i, i) \mid c+d \leq i \leq c+d+e-1\} \times E_{1} \cup\{(i, i, i) \mid c+d+e \leq i \leq l-1\} \times B_{1} \cup\left(A \backslash U_{0}\right) \times B_{1}$.

As $(0,0,0) \in B_{1} \cap C_{1} \cap D_{1} \cap E_{1}$, the set $R_{1}=\{(x(2 m+1), y(2 m+1), z(2 m+1)) \mid$ $(x, y, z) \in A\}$ is one mate of the latin bitrade $\left(R_{1}, R_{2}\right)$, where $R_{2}=\{(x(2 m+1), y(2 m+$ $1),(z+1(\bmod l))(2 m+1)) \mid(x, y, z) \in A\}$. Hence, construct the latin square $L_{1}^{h}(+0)=$ $\left(L_{1}^{h}(+0)^{\prime} \backslash R_{1}\right) \cup R_{2}$.

Let $1 \leq k \leq 2 m$.

By Lemma 3.3 the set of triples $U_{1} \times V_{1}^{f}$, where $0 \leq f \leq 2 m-1$, forms a transversal in $L_{1}^{h}(+0)$.

The latin square $L_{1}^{h}(+k)$ is constructed by $k$-prolongating $L_{1}^{h}(+0)$ down the transversals

$$
T_{1}^{f}=U_{1} \times V_{1}^{f}, \text { where } 1 \leq f \leq k,
$$

and using the completing square

$$
\{(i, j,(i+j-2 l(2 m+1)(\bmod k))+l(2 m+1)) \mid l(2 m+1) \leq i, j \leq l(2 m+1)+k-1\} .
$$

See Example A.23.

A second latin square, $L_{2}^{h}(+0)$, of order $l(2 m+1)$ will now be constructed.

$$
\begin{gathered}
L_{2}^{h}(+0)=\{(i, i, i) \mid 0 \leq i \leq c-1\} \times C_{2} \cup\{(i, i, i) \mid c \leq i \leq c+d-1\} \times D_{2} \cup \\
\{(i, i, i) \mid c+d \leq i \leq c+d+e-1\} \times E_{2} \cup\{(i, i, i) \mid c+d+e \leq i \leq l-1\} \times B_{2} \cup\left(A \backslash U_{0}\right) \times B_{2} .
\end{gathered}
$$

By Lemma 3.3 the set of triples $U_{2} \times V_{2}^{f}$, where $0 \leq f \leq 2 m-1$, forms a transversal in $L_{2}^{h}(+0)$.

Let $1 \leq k \leq 2 m$. Construct the latin square $L_{2}^{h}(+k)$ by $k$-prolongating $L_{2}^{h}(+0)$ down the transversals

$$
T_{2}^{f}=U_{2} \times V_{2}^{f}, \text { where } 1 \leq f \leq k,
$$


and using the completing square

$\{(i, j,(i+j-2 l(2 m+1)+1(\bmod k))+l(2 m+1)) \mid l(2 m+1) \leq i, j \leq l(2 m+1)+k-1\}$.

See Example A.24.

Let $0 \leq k \leq 2 m$; when $k \neq 1, L_{1}^{h}(+k)$ intersects $L_{2}^{h}(+k)$ precisely in $h$ disjoint $m$ flowers and, when $k=1, L_{1}^{h}(+1)$ intersects $L_{2}^{h}(+1)$ precisely in $h$ disjoint $m$-flowers and one other disjoint triple. See Example A.25.

Hence, the following result has been established.

Lemma 4.34. Assume $2 \leq m, 3 \leq l, 6 m+3 \leq n$ and $0 \leq h \leq 3\left\lfloor\frac{n}{2 m+1}\right\rfloor$. If $n \neq$ $l(2 m+1)+1$ then there exists a pair of latin squares of order $n$ that intersect precisely in $h$ disjoint $m$-flowers. If $n=l(2 m+1)+1$ then there exists a pair of latin squares of order $n$ that intersect in precisely $h$ disjoint $m$-flowers and one additional disjoint triple.

Let $k=1$; as above prolongate $L_{1}^{h}(+0)$ down the transversal $T_{1}^{1}=U^{1} \times V_{1}^{1}$ to achieve the latin square $L_{1}^{h}(+1)$.

Note that the set $S_{1}=\{(l(2 m+1), l(2 m+1), l(2 m+1)),(l(2 m+1)-1, l(2 m+$ $\left.1), z),(l(2 m+1)-1, y, l(2 m+1)),(l(2 m+1), y, z) \mid(l(2 m+1)-1, y, z) \in T_{1}^{1}\right\}$, forms one mate of the latin bitrade $\left(S_{1}, S_{2}\right)$, where $S_{2}=\{(l(2 m+1), l(2 m+1), z),(l(2 m+1)-$ $1, l(2 m+1), l(2 m+1)),(l(2 m+1)-1, y, z),(l(2 m+1), y, l(2 m+1)) \mid(l(2 m+1)-1, y, z) \in$ $\left.T_{1}^{1}\right\}$. Construct the latin square $L_{3}^{h}(+1)=\left(L_{1}^{h}(+1) \backslash S_{1}\right) \cup S_{2}$.

The latin squares $L_{3}^{h}(+1)$ and $L_{2}^{h}(+1)$ intersect precisely in $h$ disjoint $m$-flowers. Thus, the following result has been established.

Lemma 4.35. Assume $2 \leq m, 3 \leq l, n=l(2 m+1)+1$ and $0 \leq h \leq 3 l$, then there exists a pair of latin squares of order $n$ that intersect in precisely $h$ disjoint $m$-flowers.

Next, a pair of latin squares of order $l(2 m+1)+m \leq n<(l+1)(2 m+1), n \neq$ $l(2 m+1)+m+1$ and $(n, m) \neq(5 l+2,2)$ that intersect precisely in $3 l+1$ disjoint $m$-flowers will be constructed.

Let $m \leq k \leq 2 m, k \neq m+1$ and $(k, m) \neq(2,2)$.

Let $X_{1}=\{(i, j,(i+j-2 l(2 m+1)(\bmod k))+l(2 m+1)) \mid l(2 m+1) \leq i, j \leq$ $l(2 m+1)+k-1\}$ and $Y_{1}=\{(i, j,(i+j-2 l(2 m+1)+1(\bmod k))+l(2 m+1))$ $l(2 m+1) \leq i, j \leq l(2 m+1)+k-1\}$. Note that $X_{1} \subset L_{1}^{3 l}(+k)$ and $Y_{1} \subset L_{2}^{3 l}(+k)$ (they are the completing squares used in the $k$-prolongations).

By Lemma 4.11, as $k \neq m+1$ and $(k, m) \neq(2,2)$, there exists a pair of latin squares of order $k$ that intersect precisely in one $m$-flower. Denote this pair of latin squares by $\left\{X_{2}^{\prime}, Y_{2}^{\prime}\right\}$. Let $X_{2}=\delta^{l(2 m+1)} X_{2}^{\prime}$ and $Y_{2}=\delta^{l(2 m+1)} Y_{2}^{\prime}$.

Hence, $\left(X_{1}, X_{2}\right)$ and $\left(Y_{1}, Y_{2}\right)$ are latin biswaps. Construct the latin squares

$$
L_{4}^{3 l+1}(+k)=\left(L_{1}^{3 l}(+k) \backslash X_{1}\right) \cup X_{2} \text { and } L_{5}^{3 l+1}(+k)=\left(L_{2}^{3 l}(+k) \backslash Y_{1}\right) \cup Y_{2} .
$$

The latin squares $L_{4}^{3 l+1}(+k)$ and $L_{5}^{3 l+1}(+k)$ intersect precisely in $3 l+1$ disjoint $m$ flowers. Hence, the following result has been established. 
Lemma 4.36. Assume $2 \leq m, 3 \leq l, l(2 m+1)+m \leq n \leq l(2 m+1)+2 m, n \neq$ $l(2 m+1)+m+1$ and $(n, m) \neq(5 l+2,2)$; then there exists a pair of latin squares of order $n$ that intersect precisely in $3 l+1$ disjoint $m$-flowers.

A pair of latin squares of order $l(2 m+1)+m+1$ that intersect precisely in $3 l+1$ disjoint $m$-flowers will now be constructed.

Similarly to before, let $X_{1}=\{(i, j,(i+j-2 l(2 m+1)(\bmod m+1))+l(2 m+1)) \mid$ $l(2 m+1) \leq i, j \leq l(2 m+1)+m\}$ and $Y_{1}=\{(i, j,(i+j-2(l(2 m+1))+1(\bmod m+$ $1))+l(2 m+1)) \mid l(2 m+1) \leq i, j \leq l(2 m+1)+m\}$. Again, note that $X_{1} \subset L_{1}^{3 l}(+m+1)$ and $Y_{1} \subset L_{2}^{3 l}(+m+1)$.

By Lemma 4.11 there exists a pair of latin squares of order $m+1$ that intersect precisely in one $(m+1)$-flower. Without loss of generality the $(m+1)$-flower is comprised by the set of cells $\{(i, i, i) \mid 0 \leq i \leq m\}$. Denote this pair of latin squares by $\left\{X_{2}^{\prime}, Y_{2}^{\prime}\right\}$.

Let $X_{2}=\delta^{l(2 m+1)} X_{2}^{\prime}$ and $Y_{2}=\delta^{l(2 m+1)} Y_{2}^{\prime}$.

Hence, $\left(X_{1}, X_{2}\right)$ and $\left(Y_{1}, Y_{2}\right)$ are latin biswaps. Construct the latin squares

$L_{6}^{3 l+1}(+m+1)=\left(L_{1}^{3 l}(+m+1) \backslash X_{1}\right) \cup X_{2}$ and $L_{7}^{3 l+1}(+m+1)=\left(L_{2}^{3 l}(+m+1) \backslash Y_{1}\right) \cup Y_{2}$.

Note that the set of triples $S_{1}=\{(l(2 m+1), l(2 m+1), l(2 m+1)),(l(2 m+1)-1, l(2 m+$ $\left.1), z),(l(2 m+1)-1, y, l(2 m+1)),(l(2 m+1), y, z) \mid(l(2 m+1)-1, y, z) \in T_{1}^{1}\right\}$, forms one mate of the latin bitrade $\left(S_{1}, S_{2}\right)$, where $S_{2}=\{(l(2 m+1), l(2 m+1), z),(l(2 m+1)-$ $1, l(2 m+1), l(2 m+1)),(l(2 m+1)-1, y, z),(l(2 m+1), y, l(2 m+1)) \mid(l(2 m+1)-1, y, z) \in$ $\left.T_{1}^{1}\right\}$.

Construct the latin square $L_{8}^{3 l+1}(+m+1)=\left(L_{6}^{3 l+1}(+m+1) \backslash S_{1}\right) \cup S_{2}$.

The latin squares $L_{8}^{3 l+1}(+m+1)$ and $L_{7}^{3 l+1}(+m+1)$ intersect precisely in $3 l+1$ disjoint $m$-flowers. Hence, the following result has been established.

Lemma 4.37. Assume $2 \leq m, 3 \leq l$ and $n=l(2 m+1)+m+1$, then there exists a pair of latin squares of order $n$ that intersect precisely in $3 l+1$ disjoint $m$-flowers.

A pair of latin squares of order $5 l+2$ that intersect precisely in $3 l+1$ disjoint 2-flowers will now be constructed. Recall that there does not exist a pair of latin squares of order two that intersect precisely in one 2 -flower.

Consider the latin square of order two, $G_{1}=\{(0,0,0),(0,1,1),(1,0,1),(1,1,0)\}$.

Let $m=2$. Consider the permutation $\zeta=(12)$. Construct the latin squares $L_{1}^{3 l}(+2)$ as above using the transversals $T_{1}^{1}$ and $T_{1}^{2}$ and the completing square $\delta^{5 l} G_{1}$.

Note, the set of triples $S_{1}=\left\{(0, y, 5 l),(0,5 l, z),(5 l, y, z),(5 l, 5 l, 5 l) \mid(0, y, z) \in T_{1}^{1}\right\} \subset$ $L_{1}^{3 l}(+2)$, forms one mate of the latin bitrade $\left(S_{1}, S_{2}\right)$, where $S_{2}=\{(0, y, z),(0,5 l, 5 l)$, $\left.(5 l, y, 5 l),(5 l, 5 l, z) \mid(0, y, z) \in T_{1}^{1}\right\}$. Hence, construct the latin square $L_{9}^{3 l+1}(+2)=$ $\left(L_{1}^{3 l}(+2) \backslash S_{1}\right) \cup S_{2}$.

Next, construct the latin square $L_{10}^{3 l+1}(+2)$ by $(\zeta, \zeta)$-2-prolongating the latin square $L_{2}^{3 l}(+0)$ down the transversals $T_{2}^{1}$ and $T_{2}^{2}$ and using the completing square $\delta^{5 l} G_{1}$.

Note that the set of triples $S_{3}=\{(0, p, 5 l),(0,5 l+1, q),(5 l+1, p, q),(5 l+1,5 l+$ $\left.1,5 l) \mid(0, p, q) \in T_{2}^{1}\right\} \subset L_{10}^{3 l+1}(+2)$, forms one mate of the latin bitrade $\left(S_{3}, S_{4}\right)$, where 
$S_{4}=\left\{(0, p, q),(0,5 l+1,5 l),(5 l+1, p, 5 l),(5 l+1,5 l+1, q) \mid(0, p, q) \in T_{2}^{1}\right\}$. Construct the latin square $L_{11}^{3 l+1}(+2)=\left(L_{10}^{3 l+1}(+2) \backslash S_{3}\right) \cup S_{4}$.

The latin squares $L_{9}^{3 l+1}(+2)$ and $L_{11}^{3 l+1}(+2)$ intersect precisely in $3 l+1$ disjoint 2flowers. Hence, the following result has been established.

Lemma 4.38. Assume $3 \leq l$ and $n=5 l+2$, then there exists a pair of latin squares of order $n$ that intersect precisely in $3 l+1$ disjoint 2 -flowers.

Let $3 \leq m$. Next, a pair of latin squares of order $l(2 m+1)+2 m$, where $3 \leq l$ that intersect precisely in $3 l+2$ disjoint $m$-flowers will be constructed.

Let $X_{1}=\{(i, j,(i+j-2 l(2 m+1)(\bmod 2 m))+l(2 m+1)) \mid l(2 m+1) \leq i, j \leq$ $l(2 m+1)+2 m-1\}$ and $Y_{1}=\{(i, j,(i+j-l(2 m+1)+1(\bmod 2 m))+l(2 m+1)) \mid$ $l(2 m+1) \leq i, j \leq l(2 m+1)+2 m-1\}$. Once again, note that $X_{1} \subset L_{1}^{3 l}(+2 m)$ and $Y_{1} \subset L_{2}^{3 l}(+2 m)$.

By Lemma 4.19 there exists a pair of latin squares of order $2 m$ that intersect precisely in two disjoint $m$-flowers. Denote this pair of latin squares by $\left\{X_{2}^{\prime}, Y_{2}^{\prime}\right\}$. Let $X_{2}=$ $\delta^{l(2 m+1)} X_{2}^{\prime}$ and $Y_{2}=\delta^{l(2 m+1)} Y_{2}^{\prime}$.

Hence, $\left(X_{1}, X_{2}\right)$ and $\left(Y_{1}, Y_{2}\right)$ are latin biswaps. Construct the latin squares

$$
L_{12}^{3 l+2}(+2 m)=\left(L_{1}^{3 l}(+2 m) \backslash X_{1}\right) \cup X_{2} \text { and } L_{13}^{3 l+2}(+2 m)=\left(L_{2}^{3 l}(+2 m) \backslash Y_{1}\right) \cup Y_{2} .
$$

The latin squares $L_{12}^{3 l+2}(+2 m)$ and $L_{13}^{3 l+2}(+2 m)$ intersect precisely in $3 l+2$ disjoint $m$-flowers. Hence, the following result has been established.

Lemma 4.39. Assume $3 \leq m, 3 \leq l$ and $n=l(2 m+1)+2 m$, then there exists a pair of latin squares of order $n$ that intersect precisely in $3 l+2$ disjoint $m$-flowers.

Finally, a pair of latin squares of order $5 l+4$ that intersect in $3 l+2$ disjoint 2 -flowers will be constructed. This construction uses similar ideas to those underlying the construction used to prove Lemma 4.38 .

Consider the following pair of latin squares of order four, $G_{2}^{\prime}=\{(0,0,0),(0,1,1),(0$, $2,2),(0,3,3),(1,0,1),(1,1,0),(1,2,3),(1,3,2),(2,0,2),(2,1,3),(2,2,1),(2,3,0),(3,0,3)$, $(3,1,2),(3,2,0),(3,3,1)\}$ and $G_{3}^{\prime}=\{(0,0,0),(0,1,1),(0,2,3),(0,3,2),(1,0,1),(1,1,0)$, $(1,2,2),(1,3,3),(2,0,3),(2,1,2),(2,2,1),(2,3,0),(3,0,2),(3,1,3),(3,2,0),(3,3,1)\}$.

Let $m=2$. Consider the permutations $\zeta_{a}=\left(\begin{array}{lll}1 & 2 & 3\end{array}\right)$ and $\zeta_{b}=\left(\begin{array}{ll}1 & 4\end{array}\right)$. Construct the latin square $L_{14}^{3 l+2}(+4)$ by $\left(\zeta_{a}, \zeta_{b}\right)$-4-prolongating the latin square $L_{1}^{3 l}(+0)$ down the transversals $T_{1}^{i}$, where $1 \leq i \leq 4$ and using the completing square $G_{2}=\delta^{5 l} G_{2}^{\prime}$.

Note that the set of triples $S_{1}=\{(0, y, 5 l),(0,5 l+3, z),(5 l+2, y, z),(5 l+2,5 l+3,5 l)$, $\left.(0, v, 5 l+1),(0,5 l+1, w),(5 l, v, w),(5 l, 5 l+1,5 l+1) \mid(0, y, z) \in T_{1}^{1},(0, v, w) \in T_{1}^{2}\right\} \subset$ $L_{14}^{3 l+2}(+4)$, forms one mate of the latin bitrade $\left(S_{1}, S_{2}\right)$, where $S_{2}=\{(0, y, z),(0,5 l+$ $3,5 l),(5 l+2, y, 5 l),(5 l+2,5 l+3, z),(0, v, w),(0,5 l+1,5 l+1),(5 l, v, 5 l+1),(5 l, 5 l+1, w) \mid$ $\left.(0, y, z) \in T_{1}^{1},(0, v, w) \in T_{1}^{2}\right\}$. Construct the latin square $L_{16}^{3 l+2}(+4)=\left(L_{9}^{3 l+2}(+4) \backslash S_{1}\right) \cup S_{2}$.

Now, construct the latin square $L_{15}^{3 l+2}(+4)$ by $\left(\zeta_{b}, \zeta_{a}\right)$-4-prolongating the latin square $L_{2}^{3 l}(+0)$ down the transversals $T_{2}^{i}$, where $1 \leq i \leq 4$, and using the completing square $G_{3}=\delta^{5 l} G_{3}^{\prime}$. 
Note that the set of triples $S_{3}=\{(0, p, 5 l),(0,5 l+2, q),(5 l+3, p, q),(5 l+3,5 l+2,5 l)$, $\left.(0, s, 5 l+1),(0,5 l, t),(5 l+1, s, t),(5 l+1,5 l, 5 l+1) \mid(0, p, q) \in T_{2}^{1},(0, s, t) \in T_{2}^{2}\right\} \subset$ $L_{15}^{3 l+2}(+4)$, forms one mate of the latin bitrade $\left(S_{3}, S_{4}\right)$, where $S_{4}=\{(0, p, q),(0,5 l+$ $2,5 l),(5 l+3, p, 5 l),(5 l+3,5 l+2, q),(0, s, t),(0,5 l, 5 l+1),(5 l+1, s, 5 l+1),(5 l+1,5 l, t)$ $\left.(0, p, q) \in T_{2}^{1},(0, s, t) \in T_{2}^{2}\right\}$. Hence, construct the latin square $L_{17}^{3 l+2}(+4)=\left(L_{15}^{3 l+2}(+4) \backslash\right.$ $\left.S_{3}\right) \cup S_{4}$

The latin squares $L_{16}^{3 l+2}(+4)$ and $L_{17}^{3 l+2}(+4)$ intersect precisely in $3 l+2$ disjoint 2flowers. Hence, the following result has been proved.

Lemma 4.40. Assume $m=2,3 \leq l$ and $n=5 l+4$; then there exists a pair of latin squares of order $n$ that intersect precisely in $3 l+2$ disjoint 2 -flowers.

\subsection{Main theorem}

At the beginning of this paper necessary conditions for the disjoint $m$-flower intersection problem in latin squares were established. Through the rest of the paper pairs of latin squares that prove that these conditions are in fact sufficient have been constructed.

Theorem 1. There exists a pair of latin squares of order $n$ whose intersection is composed precisely of $x$ disjoint $m$-flowers, where $2 \leq m \leq n, 0 \leq x \leq i$ and

$$
\begin{array}{lll}
i=3 l & \text { for } & l(2 m+1) \leq n<l(2 m+1)+m \text { and } n \neq 2 m+1, \\
i=3 l+1 & \text { for } & l(2 m+1)+m \leq n<l(2 m+1)+2 m \text { and } \\
& & (m, n) \notin\{(n-1, n),(2,4)\}, \\
i=3 l+2 & \text { for } & n=l(2 m+1)+2 m, \\
i=0 & \text { for } & m=n-1, \\
i=2 & \text { for } & n=2 m+1, \text { and } \\
i=1 & \text { for } & m=2 \text { and } n=4 .
\end{array}
$$

Furthermore there does not exist a pair of latin squares of order $n$ that intersect in precisely $x>i$ disjoint $m$-flowers.

Proof. The necessary conditions for this result follow from Lemmas 4.1, 4.2, 4.4, 4.5 and 4.13. The sufficient conditions follow from Lemmas 4.3, 4.11, 4.19, 4.20, 4.23, 4.24, 4.25, $4.26,4.27,4.28,4.29,4.30,4.31,4.32,4.33,4.34,4.35,4.36,4.37,4.38,4.39$ and 4.40 .

\section{Acknowledgments}

The second author would like to thank Diane Donovan for her support and guidance. 


\section{References}

[1] Adams, P., Billington, E. J., Bryant, D. E., and Mahmoodian, E. S. The three-way intersection problem for Latin squares. Discrete Math. 243 (2002), 1-19.

[2] Bose, R. C., Shrikhande, S. S., And Parker, E. T. Further results on the construction of mutually orthogonal Latin squares and the falsity of Euler's conjecture. Canad. J. Math. 12 (1960), 189-203.

[3] Chang, Y., And Lo Faro, G. Intersection numbers of Latin squares with their own orthogonal mates. Australas. J. Combin. 26 (2002), 283-304.

[4] Chang, Y., And Lo Faro, G. The flower intersection problem for Kirkman triple systems. J. Statist. Plann. Inference 110 (2003), 159-177.

[5] Chee, Y. M. Steiner triple systems intersecting in pairwise disjoint blocks. Electron. J. Combin. 11(1) (2004), R27, 17 pp.

[6] Fu, C. M., And Fu, H.-L. The intersection problem of Latin squares. J. Combin. Inform. System Sci. 15 (1990), 89-95. Graphs, designs and combinatorial geometries (Catania, 1989).

[7] Fu, C. M., Fu, H.-L., And Guo, S. H. The intersections of commutative Latin squares. Ars Combin. 32 (1991), 77-96.

[8] Fu, C.-M., Gwo, Y.-H., And Wu, F.-C. The intersection problem for semisymmetric Latin squares. J. Combin. Math. Combin. Comput. 23 (1997), 47-63.

[9] Fu, C.-M., Huang, W.-C., Shin, Y.-H., And Yaon, Y.-J. Totally symmetric Latin squares with prescribed intersection numbers. Discrete Math. 282 (2004), 123136.

[10] Fu, H.-L. On the construction of certain types of Latin squares having prescribed intersections. PhD thesis, Department of Discrete and Statistical Sciences, Auburn University, 1980.

[11] Hoffman, D. G., And LindneR, C. C. The flower intersection problem for Steiner triple systems, vol. 149 of North-Holland Math. Stud. North-Holland, Amsterdam, 1987.

[12] Lindner, C. C., And Rosa, A. Steiner triple systems having a prescribed number of triples in common. Canad. J. Math. 27 (1975), 1166-1175.

[13] McCourt, T. A. On Defining Sets in Latin Squares and two Intersection Problems, one for Latin Squares and one for Steiner Triple Systems. PhD thesis, School of Mathematics and Physics, University of Queensland, 2010.

[14] McCourt, T. A. The intersection problem for disjoint 2-flowers in Steiner triple systems. J. Combin. Math. Combin. Comput. (in press (accepted January 2009)).

[15] Mullin, R. C., Poplove, A. L., And Zhu, L. Decomposition of Steiner triple systems into triangles. J. Combin. Math. Combin. Comput. 1 (1987), 149-174.

[16] SRinivasan, S. Disjoint intersection problem for steiner triple systems, Masters thesis, Department of Discrete and Statistical Sciences, Auburn University, 2007. 